\title{
Helical beam screen for the Future Circular Collider for hadrons injection kicker magnets
}

\author{
A. Chmielińska $\circledast^{1,2, *}$ and M. J. Barnes $\oplus^{1}$ \\ ${ }^{1}$ European Organization for Nuclear Research (CERN), CH-1211 Geneva, Switzerland \\ ${ }^{2}$ École Polytechnique Fédérale de Lausanne (EPFL), CH-1015 Lausanne, Switzerland
}

(Received 27 February 2020; accepted 13 April 2020; published 27 April 2020)

\begin{abstract}
The Future Circular Collider for hadrons (FCC-hh) is the proposed high-energy frontier particle collider, which is expected to enable proton-proton collisions at a center-of-mass energy of $100 \mathrm{TeV}$. The specifications for the FCC-hh injection kicker system are very challenging. To provide fast magnetic field rise and fall times, and low ripple during the flat-top, ferrite loaded transmission line type magnets will be used. To limit the beam coupling impedance, a suitable beam screen will be placed in the aperture of each kicker magnet. Due to issues associated with the "conventional" beam screen design, used in the injection kickers magnets of the Large Hadron Collider (LHC), such as high voltage (HV) breakdowns and yoke heating problems, we have developed a novel concept of a helical beam screen. The fundamental advantage of the new design, in comparison to the conventional beam screen, is a significant reduction of the maximum voltage induced on the screen conductors, thus a greatly reduced probability of an electrical breakdown of the beam screen. In addition, the longitudinal beam coupling impedance of the helical beam screen is optimized to reduce power deposition in the kicker magnet. Furthermore, the helical beam screen allows a reduction of the maximum transverse beam coupling impedance of the kicker system. Detailed numerical simulations, theoretical studies and experimental results demonstrate that a new design is a promising solution not only for the FCC-hh, but also for existing machines.
\end{abstract}

DOI: 10.1103/PhysRevAccelBeams.23.041002

\section{INTRODUCTION}

The FCC-hh is foreseen to extend research in high energy physics when the LHC and its high-luminosity upgrade (HL-LHC) reach their full discovery potential [1]. The FCC-hh will require a fast injection kicker system that is highly reliable and does not limit accelerator performance. To inject many trains of bunches and to achieve a high fill-factor of the FCC-hh, the kicker magnets must provide fast magnetic field rise and fall times: in addition, good flat-top quality is required. Hence, ferrite-loaded transmission line type kicker magnets, similar to those used for injection into the LHC [2], will be used in the FCC-hh. However, during operation with high beam intensity, the beam coupling impedance of the injection kicker magnets will be a critical aspect. The problems are twofold: longitudinal and transverse beam coupling impedance may drive beam instabilities, leading to beam quality degradation and hence a decrease in luminosity. Whereas,

\footnotetext{
"agnieszka.chmielinska@alumni.epfl.ch
}

Published by the American Physical Society under the terms of the Creative Commons Attribution 4.0 International license. Further distribution of this work must maintain attribution to the author(s) and the published article's title, journal citation, and DOI. the real part of the longitudinal beam coupling impedance gives rise to beam induced heating [3]. Since the circulating beam passes through the aperture of the kicker magnet, the beam induced heating, with long duration fills, causes a temperature rise of the ferrite yoke, which could exceed its Curie temperature $\left(T_{C}\right)$. In such a case, ferrites temporarily lose their magnetic properties, which could lead to a miskick of the beam: this can damage downstream equipment. The maximum FCC-hh beam current is $0.5 \mathrm{~A}$, which is lower than for LHC (0.58 A) and HL-LHC (1.12 A). The root-mean-square (RMS) bunch length for FCC-hh is $8 \mathrm{~cm}$, whereas the equivalent Gaussian for LHC is $7.55 \mathrm{~cm}$ and $9 \mathrm{~cm}$ for HL-LHC [1]. For high beam current and short bunches, if the FCC-hh injection kicker magnets were to be unshielded, a high power deposition in the magnets is expected [4]. To limit power deposition in the ferrite yoke, a suitable beam screen, placed in the aperture of each kicker magnet, will be a critical feature. The beam screen must satisfy challenging requirements of good HV performance, low beam coupling impedance, fast magnetic field rise and fall times with low ripple of the field during the flat-top, low secondary electron yield (SEY), good radiation resistance, compatibility with ultrahigh vacuum and the ability to withstand temperatures up to $350^{\circ} \mathrm{C}$ during bakeouts.

In terms of beam induced power deposition, the impedance shielding of the FCC-hh injection kicker magnets 
must be at least comparable to the effectiveness of the MKI beam screen. This would allow to use the new design also in the existing machines. However, since many injection kicker magnets are expected in the FCC, their impact on beam dynamics might be critical. To avoid coupled bunch instability in the longitudinal plane, the maximum allowable machine impedance is $2 \mathrm{MOhm}$ at $400 \mathrm{MHz}$ [5], and the threshold is less strict at other frequencies: this is discussed further in this article, together with Landau damping. To preserve longitudinal Landau damping, the upper limit for the imaginary effective longitudinal impedance is $200 \mathrm{~m} \Omega$ [1]. Detailed estimates of the impedance thresholds in the transverse planes can be found in Refs. [1,6].

Extensive optimization studies of the FCC-hh injection kicker system have been performed, in order to reduce the beam coupling impedance of the kicker magnets, while ensuring compatibility with the novel pulse power generators $[4,7]$. For the impedance shielding, the first option considered was the so-called conventional beam screen design, similar to that used for the injection kicker magnets of the Large Hadron Collider (LHC MKI) [8]. However, due to HV concerns, we have subsequently developed a novel concept of a helical beam screen. The new design allows to achieve significantly improved HV behavior and low power deposition in the kicker magnet. In addition, the helical screen design reduces the maximum transverse impedance of the kicker system, hence it is the baseline proposed for the FCC-hh.

\section{INJECTION KICKER SYSTEM}

The FCC-hh will be equipped with two injection kicker systems, to inject two counterrotating proton beams. The baseline injection energy is $3.3 \mathrm{TeV}$ [1]. In this case, the LHC is expected to be modified and used as a High Energy Booster (HEB) injector [1]. To limit the beam coupling impedance of the kicker magnets, the FCC-hh injection kicker system has been optimized, such that the total length of the kicker system is reduced from $130 \mathrm{~m}$ [9] down to $40 \mathrm{~m}$ [4]. In this case, the total deflection angle for the kicker system is $0.18 \mathrm{mrad}$, requiring an integrated field strength of $\sim 2 \mathrm{~T} \cdot \mathrm{m}$. A burst-mode transfer of 130 batches, each consisting of 80 bunches interleaved with $25 \mathrm{~ns}$ empty buckets, is envisaged. These relatively short batches are required for machine protection reasons [10]. The target filling factor of the collider is $80 \%$ (10400 bunches) [1]. Therefore fast rise and fall times are required for the magnetic field pulse. To deflect the 80 bunches of a batch, the magnetic field flat-top duration is specified to be $2 \mu \mathrm{s}$. To limit beam emittance blow-up, due to injected beam trajectory instability, the reflections and the flat-top ripple of the field must be lower than $\pm 0.5 \%$. Based on the proposed filling scheme, the magnetic field rise time must not be greater than $0.43 \mu$ s (including any field overshoot, undershoot or ripple outside of the tolerance of $\pm 0.5 \%$ ).
The magnetic field rise time requirements are significantly more challenging than for the existing LHC MKIs. For the injection into the LHC line-type modulators, i.e., pulse forming networks (PFNs), are used [11]. Each PFN is discharged through a thyratron switch to generate the current pulse for the kicker magnet. A significant drawback of the existing system is an occasional spontaneous turn-on of the thyratron switch [12], which can cause a miskick of the circulating beam. Thus, novel pulse generators based on semiconductor (SC) switches are being developed to achieve short rise times and low failure rates. However, suitably fast switching SC switches capable of both turn-on and turn-off are presently not capable of switching the required high voltage and current. Hence, a modular design, consisting of both, series and parallel SC switches, is required [13]. The Inductive Adder [7] (IA) and the Marx Generator (MG) [14] are the most promising topologies of a solid-state kicker generator. The baseline parameters of the FCC-hh injection kicker system have been selected to ensure compatibility with the aforementioned topologies. For example, as a result of the limited voltage rating of the SC switches, many series "modules" are needed to achieve the required output voltage: hence, to limit the cost and propagation delay through the stack, the output voltage must be kept at a reasonably low value [15]. To aim for a low voltage, the fast kicker magnets have low characteristic impedance, to achieve the required current, but the design must still be consistent with the field rise time requirements. Hence, the characteristic impedance of each FCC-hh injection kicker magnet is selected to be $6.25 \Omega$ [4].

To achieve the required deflection angle, several aspects have been considered in the kicker system design. Reducing the magnetic length of the system requires a higher magnetic flux density and thus a larger pulse current. For the yoke of a fast kicker magnet, a NiZn ferrite is typically used: a suitable ferrite has a saturation flux density of $\sim 0.3 \mathrm{~T}$ [16] at ambient temperature, and a lower value at higher temperatures (e.g., $0.16 \mathrm{~T}$ at $100{ }^{\circ} \mathrm{C}$ [17]). The average value of the flux density in the ferrite yoke, including fringe fields at the ends, should be kept to a value comfortably below the saturation flux density. At the same time, it is desirable to minimize the surface area of the ferrite yoke, as the ferrite is a significant contributor to outgassing, in the vacuum tank, even after a bakeout. Studies show that the outgassing increases significantly as the temperature of the ferrite rises [18]. Thus, the thickness of the ferrite yoke in the direction perpendicular to the direction of beam propagation, must be kept to a reasonable minimum. However, the thickness must be sufficient to maintain the magnetic flux density in the ferrite yoke, including end effects, to an acceptable level.

To limit the required current, the aperture dimensions should correspond to the minimum value set by beam parameters with an additional margin to provide the space for the beam screen. The minimum FCC-hh beam pipe 
diameter at injection is $32 \mathrm{~mm}$ [4]. To allow for a beam screen with a thickness of $8 \mathrm{~mm}$, as per the LHC MKI beam screen, the vertical and horizontal aperture dimensions are specified to be $48 \mathrm{~mm}$ [4]. To achieve the fast switching, MOSFETs will be used in the IA or MG: the generators repetitive design current with the present generation of high voltage and high current SiC MOSFETs, is $2.5 \mathrm{kA} \mathrm{[13].}$ Operating with the current near to the maximum allowable value, reduces the total number of magnets and pulse generators. However, in the case of a short-circuit fault, the rate of rise of current can be very high: hence, there must be an adequate margin with respect to the SC ratings, and rapid turn-off of the SCs in the event of a fault. In the proposed design, each FCC-hh injection kicker system will consist of 18 magnets, operating with a pulse current of $2.4 \mathrm{kA}$. The corresponding effective magnetic length is $31.8 \mathrm{~m}$ and the magnetic flux density, in the aperture, is $0.063 \mathrm{~T}$.

The rise and fall times of the current pulses from the IA are expected to be less then $75 \mathrm{~ns}$ [19]. Hence, the allowable propagation delay of the kicker magnet is $\sim 0.355 \mu \mathrm{s}$ [5]. In this case, the aperture length of each kicker magnet is expected to be $\sim 1.765 \mathrm{~m}$ [5]. To avoid saturation of the ferrite core, the average flux density in the ferrite of a central cell should be below $0.1 \mathrm{~T}$. To satisfy this requirement, the thickness of the ferrite is selected to be $30 \mathrm{~mm}$. The main FCC-hh injection kicker system parameters are summarized in Table I.

\section{TRANSMISSION LINE KICKER MAGNET}

A transmission line kicker magnet resembles a distributed electrical transmission line. The C-core ferrites are interleaved with high voltage capacitance plates and form individual cells of the kicker magnet [11]. The FCC-hh injection kicker magnet must satisfy challenging requirements for fast magnetic field rise time and low ripple during

TABLE I. Specifications of the FCC-hh injection kicker system.

\begin{tabular}{lc}
\hline \hline Parameter & Value \\
\hline Injection energy [TeV] & 3.3 \\
Deflection angle [mrad] & 0.18 \\
Pulse flat-top duration [ $\mu$ s] & 2 \\
Magnetic field flat-top tolerance [\%] & \pm 0.5 \\
Magnetic field rise time [ $\mu \mathrm{s}]$ & 0.43 \\
Magnet propagation delay [ $\mu \mathrm{s}]$ & 0.355 \\
Voltage [kV] & 15 \\
Current [kA] & 2.4 \\
System impedance [ $\Omega]$ & 6.25 \\
Effective magnetic length [m] & 31.8 \\
Number of modules & 18 \\
Magnetic flux density in the aperture [T] & 0.063 \\
Aperture height/width [mm] & $48 / 48$ \\
Ferrite thickness [mm] & 30 \\
\hline \hline
\end{tabular}

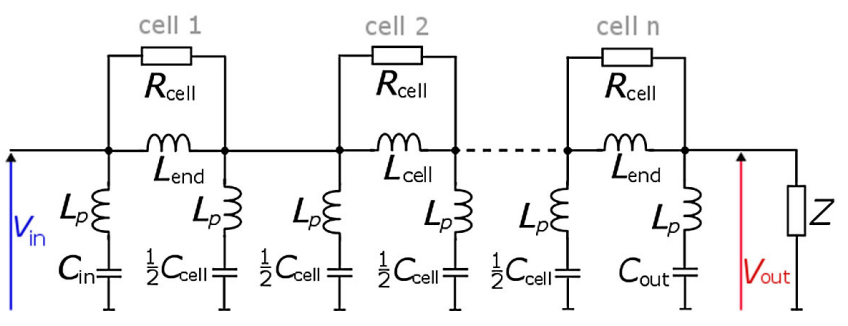

FIG. 1. The equivalent electric circuit of an n-cell transmission line kicker magnet.

the flat-top to avoid beam losses. Hence, time domain simulations have been performed in Cadence PSpice ${ }^{\circledR}$ [20], in order to select an optimal number of cells together with component parameters. For this purpose, the equivalent circuit of the transmission line kicker magnet, shown in Fig. 1, was analyzed. In Fig. 1, the characteristic impedance of the kicker magnet is $Z$. The voltages at the input and output of the kicker magnet are denoted by $V_{\text {in }}$ and $V_{\text {out }}$, respectively. The inductance and capacitance of a central cell are indicated by $L_{\text {cell }}$ and $C_{\text {cell }}$, respectively. Due to the fringe fields at both ends of the kicker magnet, the effective inductance of each end cell $\left(L_{\text {end }}\right)$ is greater than that of a central cell $\left(L_{\text {cell }}\right)$ [11]. The value of a noninductive resistor, connected in parallel with each cell, is denoted by $R_{\text {cell }}$ (Fig. 1). The capacitance value at the entrance $\left(C_{\text {in }}\right)$ and at the output $\left(C_{\text {out }}\right)$ of the kicker magnet is chosen to optimize the magnetic field pulse shape. Extensive simulations have shown that each FCC-hh injection kicker magnet must consist of at least 20 cells [5]. For such a magnet, $L_{\text {cell }}=111 \mathrm{nH}, C_{\text {cell }}=2.84 \mathrm{nF}$ and $L_{\text {end }}=126 \mathrm{nH}$. The optimized values of the other parameters of the equivalent circuit shown in Fig. 1 are given in Sec. VII F. Nevertheless, it should be noted that a beam screen placed in the aperture of the kicker magnet will influence the field response. In the model shown in Fig. 1, this effect is not taken into account. The waveform of the shielded FCC-hh injection kicker magnet is analyzed in Sec. VII.

We have developed a conceptual design of a single FCC-hh injection kicker magnet module. A 3D model of a 20-cell FCC-hh injection kicker magnet was built in CST Studio Suite ${ }^{\circledR}$ [21]: the upstream end is shown in Fig. 2. Accelerator grade CMD5005 (National Magnetics, Inc.) [17] ferrite is presently the preferred material for the yoke of the FCC-hh injection kicker magnet. In particular, accelerator grade CMD5005 is preferred over standard grade CMD5005, as accelerator grade is isostatically pressed, which provides superior real permeability to uniaxially pressed ferrite [22]. A relatively high real part of permeability is required to achieve good field homogeneity in the rectangular aperture. By contrast, a low imaginary permeability is desirable to minimize magnetic losses in the ferrite yokes. To increase the confidence in our model, the electromagnetic properties of accelerator grade 


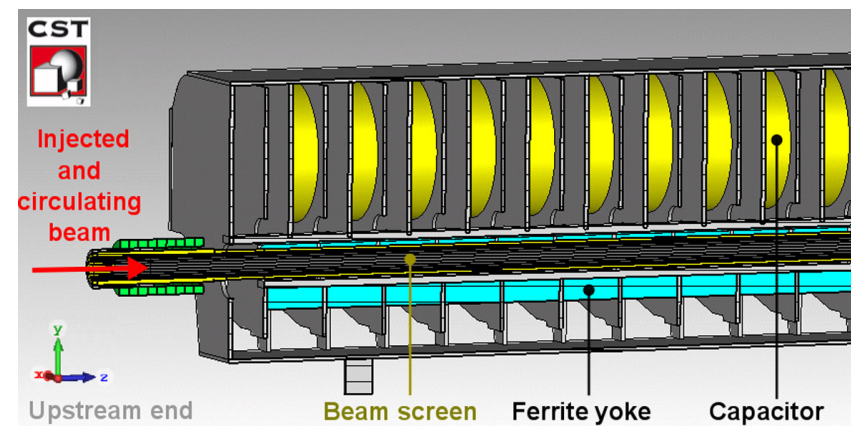

FIG. 2. A CST model of a 20-cell FCC-hh injection kicker magnet-the first 10 cells at the upstream end are shown.

CMD5005 ferrite have been characterized using shortcircuit and transmission line methods [23].

For the FCC-hh injection kicker magnet, two types of beam screens have been considered. The details of the conventional and helical beam screen designs are discussed in Sec. IV and V, respectively.

\section{CONVENTIONAL BEAM SCREEN}

Initially the so-called conventional beam screen, similar to that used in the LHC MKI, was proposed and studied for the FCC-hh injection kicker magnets. The conventional design consists of 24 straight $\mathrm{NiCr}(80 \% / 20 \%)$ screen conductors, which are $2.7 \mathrm{~mm}$ wide and $0.8 \mathrm{~mm}$ thick. The screen conductors provide a path for the beam image current, and therefore screen the ferrite yoke from the electromagnetic fields induced by the circulating beam. The screen conductors are inserted into grooved slots in the inner wall of a ceramic tube ( $99.7 \%$ alumina) that is placed in the aperture of the kicker magnet. To preserve a fast magnetic field rise time, the screen conductors are capacitively coupled to a grounded metallic cylinder at the upstream end of the kicker magnet, at which the beam enters the kicker magnet, and are directly connected to the, grounded, vacuum chamber at the other end. A CST model of the conventional beam screen developed for FCC-hh is shown in Fig. 3.

Due to the capacitive coupling at the upstream end, an open-ended resonating cavity is formed in the region where the screen conductors overlap with the outer metallic cylinder, hence the structure exhibits a resonant behavior. The resonances in the longitudinal impedance spectrum occur at the following frequencies [8]:

$$
f_{\text {conv }}^{(n)}=\frac{n c}{2 \sqrt{\varepsilon_{r, \text { eff }}}\left(L_{\text {overlap }}+\delta_{\text {fringe }}\right)},
$$

where $n$ is an integer, $c$ is the speed of light in free space, $\varepsilon_{r, \text { eff }}$ is the effective relative permittivity of the alumina between the outer metallic cylinder and the overlap of the screen conductors, $L_{\text {overlap }}$ is the length of the overlap between the outer metallic cylinder and the longest screen conductor and $\delta_{\text {fringe }}$ is the effective increase in length due to fringe fields.

Impedance studies show that it is possible to reduce the total power loss in the ferrite yoke by decreasing the overlap length [24]. This moves impedance resonant modes to higher frequencies, where there is less power in the beam spectrum [25]. As a result, an upgraded magnet, referred to as MKI8D, with $L_{\text {overlap }}=56 \mathrm{~mm}$, was installed in the LHC during the 2017-18 Year End Technical Stop (YETS) [12]. Hence, for the design of the conventional beam screen for the FCC-hh injection kicker magnets, the same overlap length was initially selected as a promising approach to limit the total power loss.

An important feature of the conventional beam screen are toroidal ferrite rings mounted around each end of the alumina tube, outside of the aperture of the kicker magnet (Fig. 3). In the LHC MKI, each set of nine rings consists of two types of alternately arranged Ferroxcube NiZn 4B3 [26] and 4M2 [27] ferrites [28]. Originally, these ferrites were mounted to damp low frequency modes [12]. However, recent studies of the longitudinal power deposition in the kicker magnet, performed on the LHC MKI,

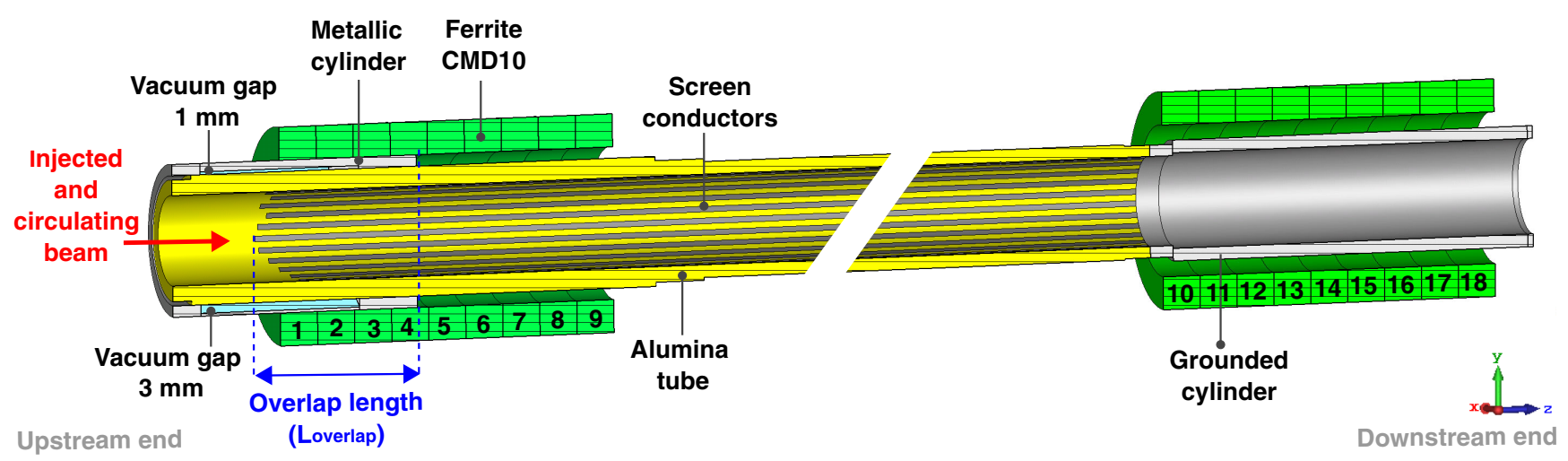

FIG. 3. A CST model of the upstream (left) and downstream (right) end of the conventional beam screen initially developed for the FCC-hh injection kicker magnets. 
have shown that ferrite rings at the upstream end can serve as a damping material to absorb beam induced power. As a result, a significantly smaller portion of beam power is dissipated in the ferrite yokes [24]. For FCC-hh, the accelerator grade CMD10 (National Magnetics, Inc.) [29] ferrite, isostatically pressed, with $T_{C} \simeq 250^{\circ} \mathrm{C}$, is a baseline material for the rings. The electromagnetic properties of the this material have been experimentally studied up to the $\mathrm{GHz}$ frequency range [23], which corresponds to the range of the beam spectrum in FCC-hh [5]: a good knowledge of the electromagnetic properties of ferrites is crucial to obtaining an accurate beam coupling impedance model and a reliable estimate of beam induced heating.

At the upstream end of the beam screen, between the alumina tube and the metallic cylinder, there is a nonsymmetric vacuum gap, which has been introduced to reduce the electric field associated with the screen conductors. The vacuum gap is $1 \mathrm{~mm}$ width at the top of the beam screen and $3 \mathrm{~mm}$ width at the bottom. The vacuum gap extends $22.5 \mathrm{~mm}$ beyond the end of the longest screen conductor at the top of the beam screen and $33.5 \mathrm{~mm}$ at the bottom. Also, as depicted in Fig. 3, to further reduce the electric field, and thus decrease the probability of electrical breakdown, the conductive wires have graded lengths at the upstream end. The vacuum gap and graded lengths were introduced in 2013 [30] and are part of the so-called postLS1 design. In the LHC, all the injection kicker magnets have such a design. For all LHC MKIs, except the MKI8D, $L_{\text {overlap }}=130 \mathrm{~mm}$.

\section{A. High voltage issues of MKI beam screen}

During magnetic field rise and fall, a significant voltage is induced on the beam screen conductors. Figure 4 shows a cross-sectional view of the beam screen. The voltage induced $\left(V_{i}\right)$ on the $i$ th screen conductor is:

$$
V_{i}=\frac{d \Phi}{d t}=\frac{d B}{d t}\left(d+R-y_{i}\right) L
$$

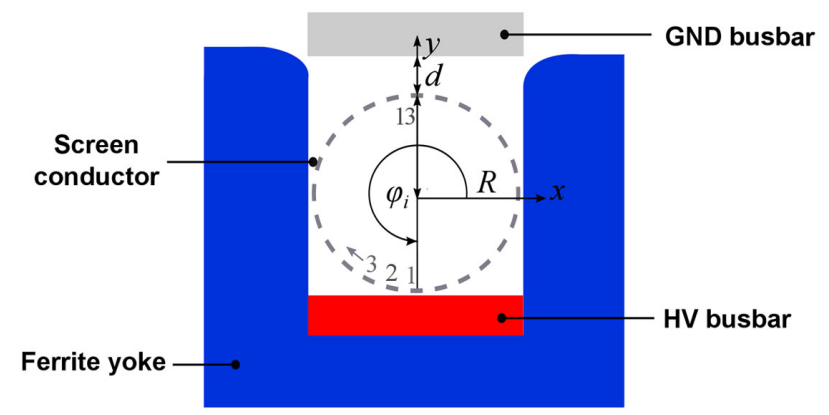

FIG. 4. Schematic cross section of a beam screen inside the aperture of the kicker magnet. The dashed circle represents the position of each screen conductor, either straight or helical, inside the alumina tube.

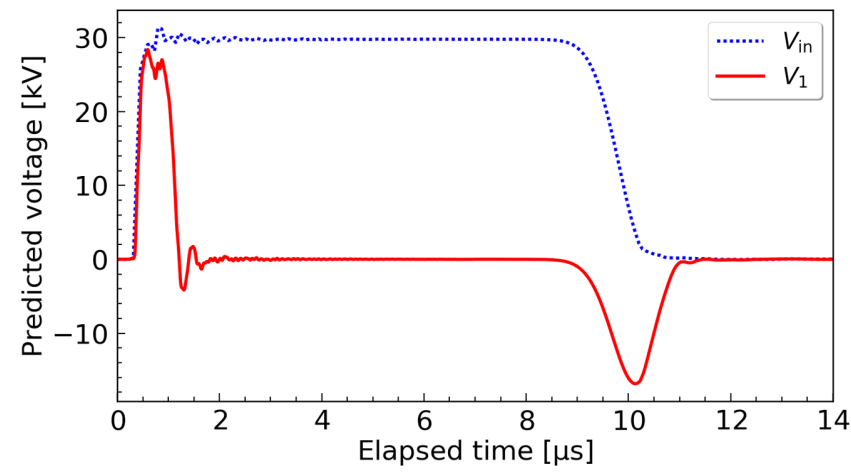

FIG. 5. Voltage at the input of the kicker magnet $\left(V_{\text {in }}\right)$ and voltage of screen conductor adjacent to HV busbar $\left(V_{1}\right)$ for $60 \mathrm{kV}$ PFN voltage [30].

where $L$ is the length of the screen conductor within a uniform magnetic field $B$ inside the aperture of the kicker magnet, $R$ is the beam screen radius, $d$ is the distance from the ground (GND) busbar to the beam screen, and $y_{i}$ is the coordinate of the $i$ th screen conductor on the $y$-axis.

The voltage is induced during the rise and fall of the magnetic field, when the time derivative of the magnetic field is nonzero. In particular, the induced voltages are positive during magnetic field rise and negative during magnetic field fall. Figure 5 shows the voltage at the input of the kicker magnet (blue dotted curve) and the voltage induced on a single screen conductor adjacent to the $\mathrm{HV}$ busbar (red solid curve).

The highest voltage is induced on the screen conductor furthest from the GND busbar, which is the conductor adjacent to the HV busbar (\#1 in Fig. 4), whereas the lowest voltage is induced on the screen conductor closest to the GND busbar (\#13): Figure 6 shows the maximum induced voltage for each of the 24 screen conductors (red curve with circular markers), as well as the difference in the maximum induced voltage between two neighboring screen conductors (green curve with triangular markers).

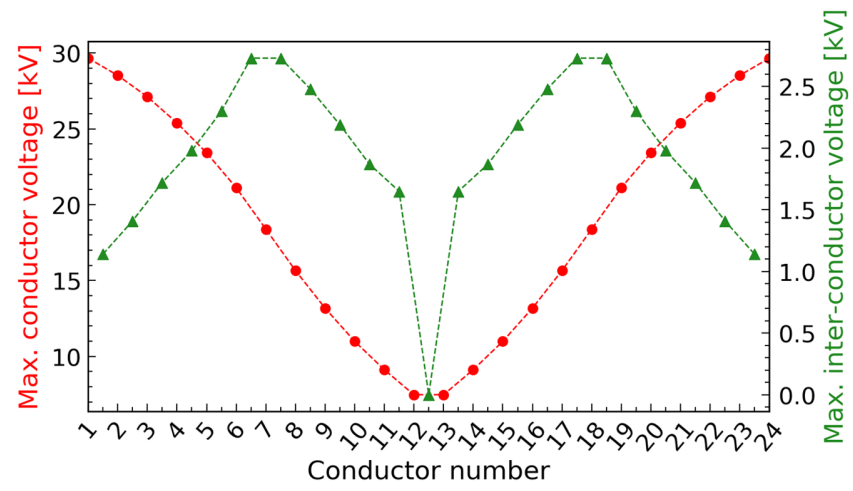

FIG. 6. Maximum conductor (circular markers) and interconductor (triangular markers) voltages for $60 \mathrm{kV}$ PFN voltage [30]. 
Voltage breakdowns, along the surface of the alumina tube, between neighboring screen conductors or between screen conductors and the metallic cylinder, are a concern during operation of the LHC MKI and issues have occurred $[30,31]$. Hence, extensive studies were carried out to optimize the HV performance of the LHC MKI $[30,32,33]$.

In 2012, a total of 5 conductors closest to the HV busbar of one MKI were removed allowing to sustain $50 \mathrm{kV}$ PFN voltage, however the missing screen conductors increased total deposited power by a factor of $\sim 1.5$ with respect to 24 screen conductors [30], which was unacceptable. Finally, the so-called "post-LS1" design permitted installation of 24 solid screen conductors: the required PFN voltage, without flashover, was achieved by introducing the aforementioned vacuum gap and grading of the screen conductor lengths at the upstream end (see Fig. 3) [30].

\section{B. Heating problems of MKIs}

During Run 2 (2015-2018) operation, the measured temperatures at the upstream end of each LHC MKI were consistently higher then those at the downstream end [34]. The electromagnetic simulations predicted that the power deposition in the LHC MKI is nonuniform [34]. Impedance studies showed that it is possible to reduce the total power loss by shortening the metallic cylinder $[34,35]$. Hence, a reduction of $L_{\text {overlap }}$ from $130 \mathrm{~mm}$ to $56 \mathrm{~mm}$ was proposed and a prototype magnet (MKI8D), with this overlap, was installed in the LHC in YETS 2017/18. In comparison with the post-LS1 design, the power in the upgraded magnet is expected to be deposited mainly in the ferrite rings at the upstream end, leading to a significant reduction of the heat load in the ferrite yokes [35]: this expectation was confirmed during LHC operation [36]. Nevertheless, for Run 3 with High Luminosity type beams, unless other mitigating measures are taken, the power loss in the kicker magnets will be unacceptably high, therefore a water cooling circuit for the ferrite rings has been developed [37].

\section{NEW CONCEPT OF A HELICAL BEAM SCREEN}

Due to the HV issues associated with the conventional beam screen design, we have proposed and studied the new concept of a helical beam screen. In this design, the screen conductors are not straight, but each of them is twisted along the length of the alumina tube. To preserve a fast magnetic field rise time, the screen conductors are capacitively coupled at the upstream end of the magnet.

Let us denote $N_{\text {turn }}$ as the number of turns of a helical conductor along the length $(L)$ of the aperture of the kicker magnet. The length of the helical conductor inside the magnet aperture is:

$$
s=\sqrt{L^{2}+\left(2 \pi R N_{\mathrm{turn}}\right)^{2}} .
$$

As each conductor extends from the downstream to the upstream end of the beam screen, over a distance $L_{\text {total }}$, and assuming a continuous helix, the total number of turns is: $N_{\text {total }}=\frac{L_{\text {total }}}{L} N_{\text {turn }}$. The pitch of the helix is the distance which 1 turn of the helix spans: $p=\frac{L}{N_{\text {turn }}}$.

\section{A. High voltage analysis}

For the helical beam screen, the coordinates of a point of the $i$ th helical screen conductor can be described as:

$$
\left\{\begin{array}{l}
x_{i}(\theta)=R \cos \left(\theta+\phi_{i}\right) \\
y_{i}(\theta)=R \sin \left(\theta+\phi_{i}\right) \\
z_{i}(\theta)=\frac{p \theta}{2 \pi} ; \theta \in\left[0 ; 2 \pi N_{\text {turn }}\right],
\end{array}\right.
$$

where $\phi_{i}$ is the initial angle of the $i$ th screen conductor at the start of the cross section (see Fig. 4). Assuming 24 conductors, $\phi_{i}=\frac{3}{2} \pi-\frac{2 \pi(i-1)}{24}$. The distance from the GND busbar for the $i$ th screen conductor changes along the longitudinal direction and can be expressed as:

$$
f_{i}(\theta)=d+R-y_{i}(\theta) .
$$

Note: $f_{i}(\theta)$ does not depend on $x_{i}$. To compute the total induced voltage, for $\zeta=2 \pi N_{\text {turn }}$, let $k=\frac{d B}{d t} \frac{p}{2 \pi}$, hence:

$$
\begin{aligned}
V_{i} & =k \int_{0}^{\zeta} f_{i}(\theta) d \theta=k \int_{0}^{\zeta}\left(d+R-R \sin \left(\theta+\phi_{i}\right)\right) d \theta \\
& =\frac{d B}{d t}(d+R) L-k R\left(\cos \left(\phi_{i}\right)-\cos \left(\phi_{i}+\zeta\right)\right) .
\end{aligned}
$$

It can be shown that $V_{i}$ is independent of $\phi_{i}$ when $N_{\text {turn }}$ is an integer. In this case, the total induced voltage on each screen conductor is the same: $V_{i}=\frac{d B}{d t}(d+R) L$. This is approximately one-half of the worst case voltage induced on a straight screen conductor adjacent to the HV busbar: $V_{1}=\frac{d B}{d t}(d+2 R) L$. Also, for straight conductors, there can be a significant difference in the induced voltage between adjacent conductors, especially for $\phi_{i} \sim 0$ and $\phi_{i} \sim \pi$. In contrast, for the helical design, there is no or little difference at the upstream end for $N_{\text {turn }}$ equal to an integer value. Although there could be a small difference in induced voltages due to fringe fields at each end of the kicker magnet, a helical beam screen is expected to provide significantly improved HV performance, in comparison with straight conductors.

\section{B. Impact of the number of turns}

The effect of the helical beam screen on longitudinal impedance has been analyzed for the first time using the CST Wakefield Solver [38]. To accurately represent small features of the beam screen, a high mesh density is 


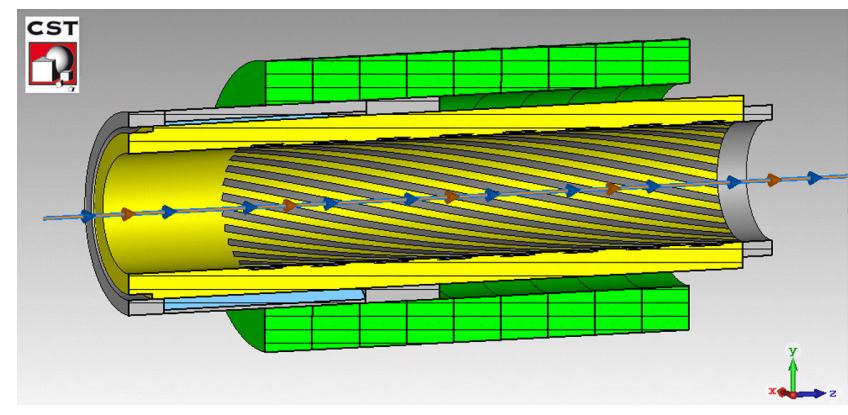

FIG. 7. Helical beam screen (cut-down model).

required. Also, a sufficiently long simulation time is necessary to achieve good frequency resolution. In Ref. [5], several approaches have been proposed to simplify simulations of the conventional beam screen in order to reduce the computation time. In particular, the predictions for the kicker magnet with the beam screen (full model), just the beam screen (simplified model) and only the upstream end of the beam screen (cut-down model) have been analyzed. For all three models, a very good agreement has been achieved when comparing the frequency and the magnitude of the fundamental harmonic in the longitudinal impedance spectrum [5]. This demonstrates a high efficiency of electromagnetic shielding of the ferrite yoke, from the beam, and shows that the kicker magnet can be excluded from the simulations for calculating the longitudinal impedance. The full model of the kicker magnet with the conventional beam screen requires approximately 2 weeks of computation on a high performance cluster (16 cores, 128 GB RAM), while the cut-down model is analyzed in 48 hours. For the helical models, due to the lack of left-right symmetry, which is present in the conventional design, the computation time doubles. Hence, the helical design was analyzed with a simplified or cutdown model (see Fig. 7). In particular, a nonsymmetric vacuum gap, ungraded length of screen conductors and $L_{\text {overlap }}=56 \mathrm{~mm}$ were considered.

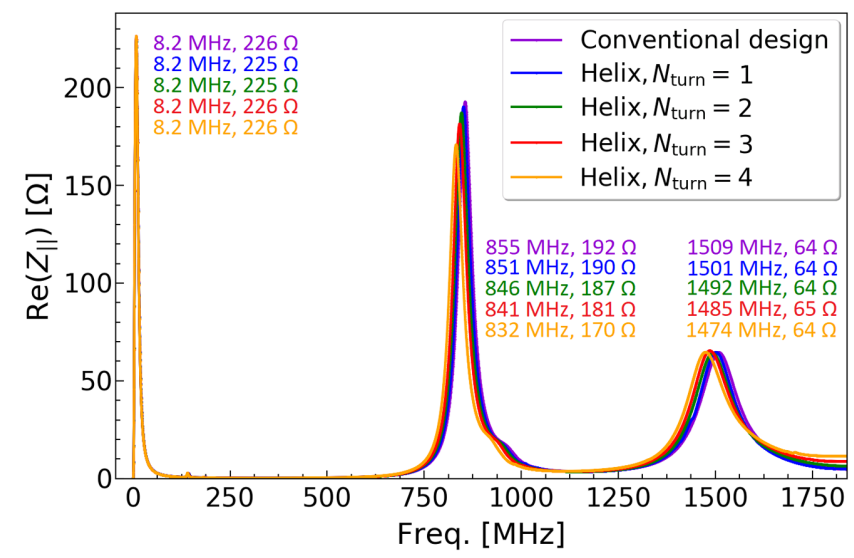

FIG. 8. Real part of the longitudinal impedance of the helical and conventional beam screen design (cut-down models).
TABLE II. Parameters of the helical beam screen.

\begin{tabular}{lccccc}
\hline \hline$N_{\text {turn }}$ & $N_{\text {total }}$ & $x_{\text {overlap }}[\mathrm{mm}]$ & $\frac{x_{\text {overlap }}}{L_{\text {overlap }}}$ & $\frac{f_{\text {conv }}^{(1)}}{f_{\text {helix }}^{(1)}}$ & $\frac{f_{\text {conv }}^{(2)}}{f_{\text {helix }}^{(2)}}$ \\
\hline 1 & 1.18 & 56.09 & 1.002 & 1.004 & 1.005 \\
2 & 2.35 & 56.36 & 1.006 & 1.011 & 1.011 \\
3 & 3.53 & 56.81 & 1.014 & 1.015 & 1.015 \\
4 & 4.70 & 57.43 & 1.025 & 1.027 & 1.023 \\
\hline \hline
\end{tabular}

A comparison of the longitudinal impedance spectrum of the conventional design with the helical design for different numbers of turns is shown in Fig. 8. For the configurations analyzed, the fundamental resonance, due to capacitive coupling, occurs between $832 \mathrm{MHz}$ and $855 \mathrm{MHz}$, while the second harmonic occurs between $1474 \mathrm{MHz}$ and $1509 \mathrm{MHz}$. The low frequency mode at $\sim 8.2 \mathrm{MHz}$ is attributed to a resonance between the inductance of the upstream ferrite rings and the coupling capacitance [5]. In particular, this mode does not contribute to the total power loss, as it is far from the first beam harmonic of $40 \mathrm{MHz}$.

The parameters of the helical conductors are listed in Table II. The length of a single screen conductor in the overlap region is denoted by $x_{\text {overlap. For helical screen }}$ conductors $x_{\text {overlap }}>L_{\text {overlap}}$, whereas for straight ones $x_{\text {overlap }}=L_{\text {overlap. }}$ Remark: the $n$th harmonic of the fundamental mode in the longitudinal impedance spectrum of the conventional design is denoted by $f_{\text {conv }}^{(n)}$ [see Eq. (1)], while for the helical design it is denoted by $f_{\text {helix }}^{(n)}$.

The simulation results show that $f_{\text {helix }}^{(n)}$ is determined by the length $x_{\text {overlap }}$. This becomes evident when comparing $f_{\text {conv }}^{(n)} / f_{\text {helix }}^{(n)}$ with respect to $x_{\text {overlap }} / L_{\text {overlap: }}$ the two ratios are in reasonably good agreement. Hence, Eq. (1) should be generalized by using $x_{\text {overlap }}$ instead of $L_{\text {overlap }}$ when the screen conductors are not straight. As $x_{\text {overlap }}$ increases with the number of turns, the main harmonics shift to lower frequencies (see Fig. 8). In general, this is expected to increase the total power loss, although the shift in frequency is marginal. To address this, we could propose to use a helical beam screen, with an integer number of turns over the effective length of the kicker aperture, with straight conductors in the overlap region.

\section{OPTIMIZATION OF THE HELICAL BEAM SCREEN}

The baseline design of a helical beam screen for the FCC-hh injection kicker magnets is presented in Fig. 9(a). Extensive simulations have been carried out to select an optimal overlap length [5]. Studies show that the total power dissipated in the kicker magnet can be significantly reduced with an overlap length of $44 \mathrm{~mm}$ [5], by reducing the length of the screen conductors at the upstream end of the magnet. Moreover, it has been demonstrated that a further decrease in the overlap length is not permitted, as it 


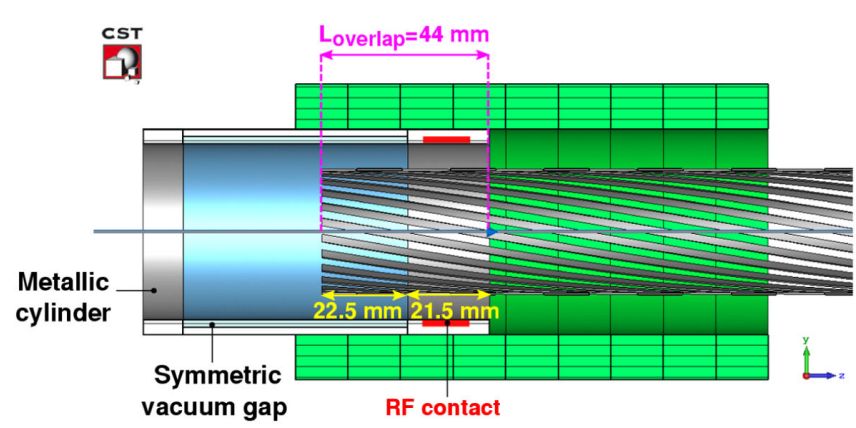

(a) Baseline design.

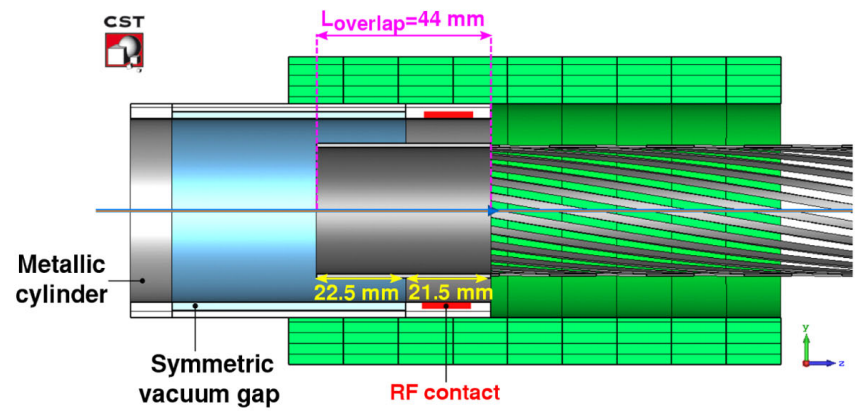

(b) Alternative design

FIG. 9. Optimized models of the helical beam screen with a symmetric vacuum gap and $L_{\text {overlap }}=44 \mathrm{~mm}$. In a baseline design, the screen conductors have ungraded lengths [Fig. 9(a)], whereas in an alternative design all screen conductors are connected in the overlap region [Fig. 9(b)].

would shift the low frequency mode closer to beam harmonics of $40 \mathrm{MHz}$, for $25 \mathrm{~ns}$ bunch spacing.

Between the outside of the alumina tube and the metallic cylinder, where there is no vacuum gap deliberately introduced, the outside alumina tube must be metallized to prevent $\mathrm{HV}$ issues here. A radio frequency (rf) contact between the metallization and the metallic cylinder ensures good electrical contact for the beam image current. Hence, to provide a metallized length of $21.5 \mathrm{~mm}$ for the $\mathrm{rf}$ contact between the metallic cylinder and the alumina tube, as in the conventional design [5], the overlap length has been reduced by shortening the screen conductors from the left side instead of cutting the metallic cylinder at the right side: this ensures the same length of exposure of the ferrite rings to the beam is achieved as in the upgraded MKI8D. Hence, the power deposition distribution in the kicker magnet with the proposed helical beam screen will be similar to the conventional design [5].

In addition, since the asymmetric vacuum gap introduces a quadrupolar component of transverse impedance [5], the helical design uses a symmetric vacuum gap. For the helical design with an integer number of turns, the total induced voltage, on each conductor, is nominally equal to that of a conductor at a distance of $d+R$ from the GND busbar in the conventional beam screen (see Fig. 4). Hence, for HV reasons [32], the vacuum gap used for the helical design can be equal to that at the vertical centre of the conventional design, i.e., $2 \mathrm{~mm}$. As in the conventional design, the vacuum gap extends beyond all the screen conductors for a distance of $22.5 \mathrm{~mm}$ [see Fig. 9(a)], to reduce the electric field [32].

For the helical beam screen, the total induced voltage on every screen conductor is the same, hence interconductor voltage breakdowns are expected to be eliminated. Moreover, the induced voltage is significantly lower in comparison with the maximal induced voltage on the straight screen conductors in the conventional design (see Sec. V). For this reason, ungraded screen conductors have been proposed in the baseline model of the helical beam screen for the FCC-hh injection kicker magnets.

As the total induced voltage on each helical conductor is nominally identical, and hence eddy currents should not circulate between conductors, a design with all helical conductors connected together in the overlap region has been also investigated. This alternative design is presented in Fig. 9(b). The proposed idea is very promising for future machines, e.g., the FCC-hh: if there was a "switch" to connect all screen conductors to the beam pipe once the injection is complete, there would be a continuous path for the beam image current and hence low beam coupling impedance. Whereas, for unconnected screen conductors, a switch would be necessary for each screen conductor, which would increase cost and complexity.

\section{A. Longitudinal beam coupling impedance}

The longitudinal impedance predictions for the baseline design with various numbers of turns, and the alternative model with 3 turns, are presented in Fig. 10. The simulations have been performed in the CST Wakefield Solver [38]. As can be observed, the proposed modifications allow us to shift the fundamental harmonics of the impedance resonances to higher frequencies in comparison with the initial design of the conventional beam screen. The helical design with screen conductors all connected together in the overlap region shifts the main harmonic to an even higher frequency. As shown in Sec. V, this is due to the length of the overlap being reduced from $x_{\text {overlap }}$ to $L_{\text {overlap }}$.

\section{B. Beam induced heating}

The heat load dissipated in an accelerator component is caused by the coupling of the beam spectrum $\left(\hat{\lambda}_{\text {beam }}\right)$ to the real part of the longitudinal impedance $\left(Z_{\|}\right)$and can be calculated using the following formula [39]:

$\Delta P=\left(Q_{b} N_{b} f_{0}\right)^{2} \sum_{p=-\infty}^{p=+\infty}\left|\hat{\lambda}_{\text {beam }}\left(p \omega_{0}\right)\right|^{2} \operatorname{Re}\left[Z_{\|}\left(p \omega_{0}\right)\right]$,

where $Q_{b}$ is the bunch charge, $N_{b}$ is the total number of bunches, and $f_{0}$ is the revolution frequency, $\omega_{0}=2 \pi f_{0}$. To calculate the FCC-hh beam spectrum, a baseline filling 


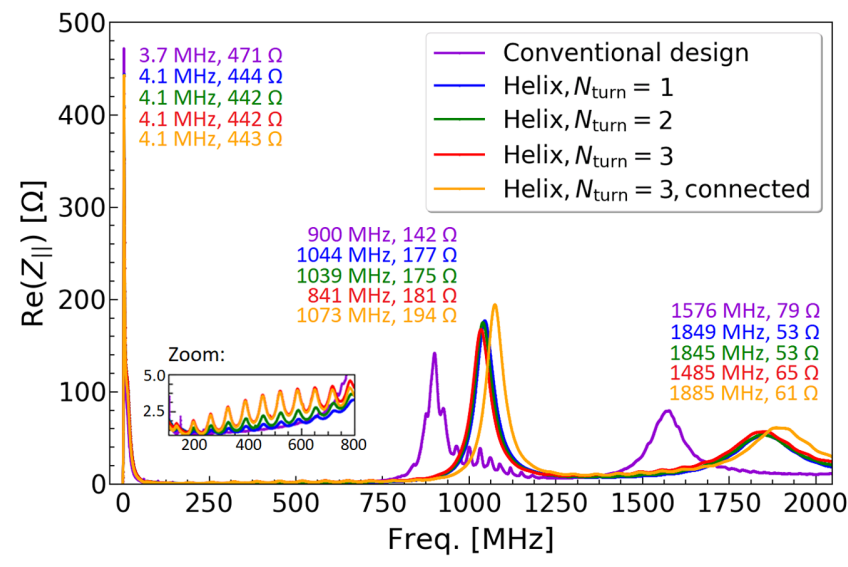

FIG. 10. Real part of the longitudinal impedance of the helical beam screen with a different number of turns, a symmetric vacuum gap, $L_{\text {overlap }}=44 \mathrm{~mm}$ and ungraded screen conductors, in comparison with the initial design of the conventional beam screen and $L_{\text {overlap }}=56 \mathrm{~mm}$ (simplified models).

scheme has been considered (see Sec. II). Assuming the nominal FCC-hh beam parameters [1], i.e., $1.0 \times 10^{11}$ protons per bunch, 10400 bunches and RMS bunch length of $0.08 \mathrm{~m}$, the total power loss for the helical models is between $31 \mathrm{~W}$ (for 1 turn) and $36 \mathrm{~W}$ (for 3 turns), while for the FCC-hh conventional design we expect $32 \mathrm{~W}$. For helical models, a significant contribution to the total predicted power loss is between $100 \mathrm{MHz}$ and $800 \mathrm{MHz}$ (see the zoom in Fig. 10), where no resonances are expected to occur. Hence, these low- $Q$ resonances are thought to be an artefact of the simulations for helixes with an increased number of turns, rather than real effects.

In all cases, the total power loss is lower than for the upgraded LHC MKI8D with nominal LHC parameters (37 W, not scaled) [25]. Moreover, similar power deposition distribution will occur in the FCC-hh injection kicker magnets as in the upgraded LHC MKI8D [5]. Since the upgraded magnet has not limited LHC operation [12], we conclude that heating of the FCC-hh injection kickers is acceptable as well. The longitudinal impedance of the helical beam screen has been experimentally verified. For this purpose, a prototype alumina tube with helical screen conductors applied using a silver paint, has been installed in a spare LHC MKI for beam coupling impedance measurements. The results are discussed in Sec. VIII.

\section{Impact on longitudinal beam dynamics}

The contribution of the FCC-hh injection kicker magnets with a beam screen to the threshold $\left(R_{\|}\right)$for the longitudinal coupled bunch instability has been evaluated. For a fixed bunch length, the threshold for the longitudinal coupled bunch instability is a function of the resonant frequency [40]. In FCC-hh, the lowest threshold is found at the frequency of $400 \mathrm{MHz}\left(R_{\|} \simeq 2 \mathrm{M} \Omega\right)$ [5,41]: at higher or lower frequencies the probability of driving an instability is smaller. Importantly, the resonances in the longitudinal impedance spectra of conventional or helical beam screens (see Fig. 10) are a few orders of magnitude below this threshold. For 18 kicker magnets, the maximal contribution to the threshold is $\sim 0.05 \%$ at the frequency of the fundamental mode ( $1000 \mathrm{MHz})$ [5]. Hence, we conclude that FCC-hh injection kicker magnets, either with the conventional or helical beam screen, are unlikely to drive a longitudinal coupled bunch instability.

In FCC-hh, to preserve longitudinal Landau damping, the upper limit for the imaginary effective longitudinal impedance is $200 \mathrm{~m} \Omega$ : to provide a safety margin, this is a factor of two higher than for LHC (100 m $\Omega)$ [1]. To estimate the impedance contribution of shielded FCC-hh injection kicker magnets to the given impedance budget, we analyzed the imaginary effective longitudinal impedance of the conventional and of the helical models, assuming Gaussian and parabolic spectral power densities [5]. For 18 kicker magnets with the conventional beam screen, the contribution is $\sim 3 \%$ of the $200 \mathrm{~m} \Omega$. In the worst case, for the helix with 3 turns, the contribution is $\sim 6 \%$. Therefore, the FCC-hh injection kicker magnets contribute to the total impedance budget at an acceptable level. Also, the analysis shows that reduction of the total length of the kicker magnets from $\sim 130 \mathrm{~m}$ to $\sim 40 \mathrm{~m}$ (see Sec. II) is important for minimizing their impact on longitudinal beam dynamics.

\section{Transverse beam coupling impedance}

Extensive simulations were carried out to analyze the transverse impedance of the helical beam screen. For this purpose, the CST Wakefield Solver [38] and the CST Eigenmode Solver [42] were used. Due to computational constraints, we performed simulations of the beam screen without the kicker magnet (simplified model).

In the CST Wakefield Solver, to analyze resonances with high $Q$-factors, the wakelength necessary to compute the impedance spectrum with a good frequency resolution requires several weeks of computation. Otherwise, the impedance calculated from a partially decayed wake potential brings a significant risk of underestimating the shunt impedance of the narrow resonant modes [43]. Let us remark: the helical beam screen is asymmetric. Hence, in the CST Wakefield Solver, we first evaluated the constant term, when both the source and the test particle are on the nominal beam axis. To calculate the dipolar impedance, we displaced the beam transverse location, while leaving the transverse position of the wake integration path at the nominal beam axis. To obtain the quadrupolar impedance, we displaced the transverse location of the wake integration path, while leaving the beam transverse location at the nominal beam axis.

To validate results, another set of simulations has been performed using the CST eigenmode solver. For each mode found in the CST wakefield solver, we performed 
eigenmode simulations on a very narrow frequency range (i.e., $10 \mathrm{MHz}$ ). To properly model the behavior of ferrites, we allowed the program to interpolate material properties within the selected frequency range. To disentangle the dipolar and the quadrupolar modes, we used the method described in Ref. [44]. Both the dipolar and the quadrupolar impedances have been computed as the sum of the eigenmode solutions using the resonator model [45]. In the present article, the main focus is on the helical beam screen. More detailed benchmark concerning the conventional design can be found in Ref. [5].

The real part of the dipolar horizontal transverse impedance of the helical beam screen with 3 turns in the aperture, a symmetric vacuum gap and $L_{\text {overlap }}=44 \mathrm{~mm}$ is shown in Fig. 11: a full decay of wake potential has been achieved in the wakefield simulation (using a $500 \mathrm{~m}$ wakelength), hence this should be a representative prediction. The wakefield solver is run first to identify peaks of interest, and then the eigenmode solver is used. Despite the complexity of the models, very good agreement has been achieved in the predicted resonant frequencies using both the wakefield and the eigenmode solvers. However, discrepancies in the magnitude of the resonances are not understood, but we consider them as acceptable. In Fig. 11, the resonances occur every $44 \mathrm{MHz}$ and are observed approximately at the following frequencies: $61 \mathrm{MHz}$, $105 \mathrm{MHz}, 149 \mathrm{MHz}, 193 \mathrm{MHz}, 237 \mathrm{MHz}, 281 \mathrm{MHz}$, $325 \mathrm{MHz}$. The resonances are odd-integer multiples of $\sim 21 \mathrm{MHz}$, which is similar to the $20 \mathrm{MHz}$ of the conventional design (see grey curve in Fig. 12) [5], except the first harmonic does not appear. The frequency of the fundamental harmonic might be slightly different in comparison with the conventional design, as the length of the screen conductors is different due to the shorter overlap length and the twist of the helical screen conductors. The strongest mode is observed at $193 \mathrm{MHz}$ (Fig. 11), which is not the fundamental harmonic: this attribute of the helical beam screen is beneficial and will be discussed further.

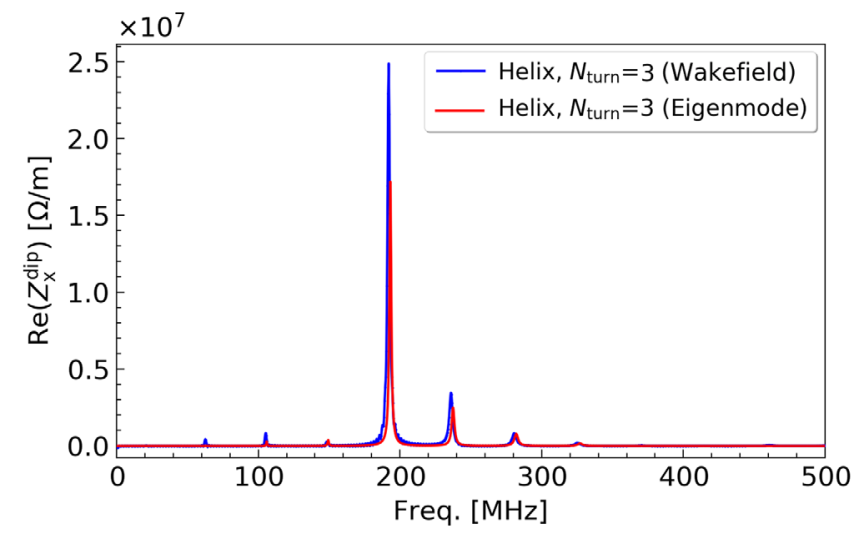

FIG. 11. Real part of the horizontal dipolar transverse impedance of a helix with 3 turns, a symmetric vacuum gap and $L_{\text {overlap }}=44 \mathrm{~mm}$.

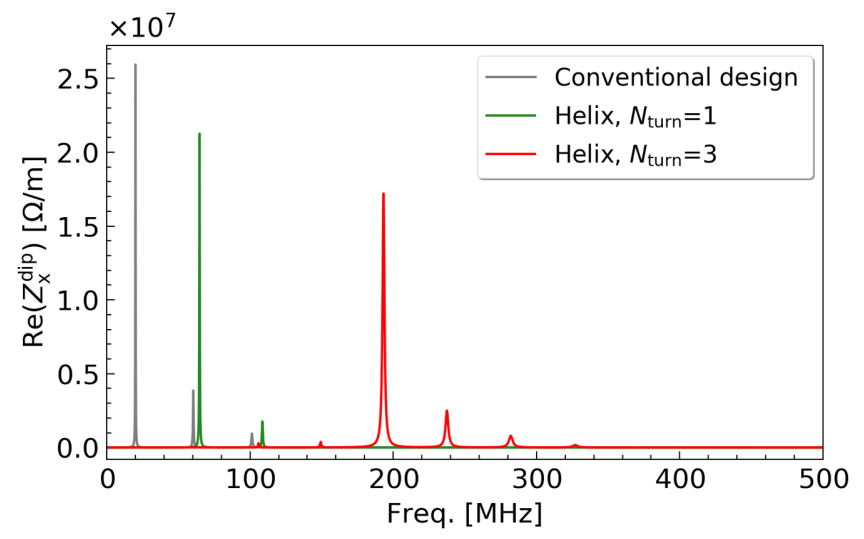

FIG. 12. Real part of the horizontal dipolar transverse impedance of the helix with 1 and 3 turns, a symmetric vacuum gap and $L_{\text {overlap }}=44 \mathrm{~mm}$, in comparison with the initial design of the conventional beam screen (eigenmode solver).

The magnitude of the strongest real mode is $\sim 18 \mathrm{M} \Omega / \mathrm{m}$ (eigenmode solver) or $\sim 24 \mathrm{M} \Omega / \mathrm{m}$ (wakefield solver). Note, for the vertical plane, the same conclusions apply. In addition, studies show that for the helical beam screen with a symmetric vacuum gap, the quadrupolar component of the transverse impedance is zero. In this regard, both of the solvers agree [5].

A significant advantage of the helical beam screen is the possibility to tune the resonant frequency of the transverse modes. Figure 12 presents the real part of the dipolar horizontal impedance for a helical beam screen with 1 and 3 turns, a symmetric vacuum gap and $L_{\text {overlap }}=44 \mathrm{~mm}$, in comparison with the initial design of the conventional beam screen and $L_{\text {overlap }}=56 \mathrm{~mm}$, evaluated using the eigenmode solver. With straight screen conductors, the first mode occurs at $20 \mathrm{MHz}$ and it has the highest shunt impedance $(\sim 26 \mathrm{M} \Omega / \mathrm{m})$. For a 1 turn helix, the strongest resonance is at $64 \mathrm{MHz}(\sim 21 \mathrm{M} \Omega / \mathrm{m})$, while for a 3 turn helix it is at $193 \mathrm{MHz}(\sim 18 \mathrm{M} \Omega / \mathrm{m})$. Hence, the number of turns determines the frequency of the strongest mode and the observed change in frequency of the strongest mode is significant.

\section{E. Transverse impedance budget considerations}

To evaluate the impedance impact of 18 kicker magnets on transverse beam stability in the FCC-hh, their contribution has been taken into account in the entire transverse impedance model of the machine $[6,46]$. With the given impedance budget for a coupled bunch instability in the transverse plane, only the helical beam screen could be used in an FCC-hh injection system, composed of 18 kicker magnets, by tuning the number of turns [46]. Based on the results presented above, several approaches can be proposed to tune the frequencies of the main resonances, i.e., different number of turns inside the magnet aperture, the same number of turns inside the magnet aperture but more 
turns outside of the aperture, or a different length of the beam screen outside of the aperture. Otherwise, the kicker magnets are mechanically, nominally identical. However, to evaluate the beam coupling impedance, each modified beam screen should be analyzed separately. Importantly, shifting the resonant frequency for many kicker magnets is not possible with the conventional design, as it would require the overlap of some kickers to be changed and hence it would have destructive impact on the longitudinal impedance and would cause excessive heating. Whereas, changing the number of turns of the helical design has little influence upon the magnitude and frequency of the resonant modes (Fig. 8).

\section{FIELD RESPONSE OF THE KICKER MAGNET}

The beam screen in the aperture of the kicker magnet is expected to influence the magnetic field rise time and the field flat-top quality, as it causes a significant frequency dependence of the kicker magnet inductance [47]. In this section, such a dependence is quantified using the finite element method (FEM) algorithms implemented in Opera-2D [48].

\section{A. General considerations}

As described in Sec. IV, the beam screen conductors are inserted in the inner surface of the alumina tube to screen the ferrite yoke from the electromagnetic fields induced by the circulating beam, which could cause an excessive heating of the ferrite yoke. The alumina chambers allow the penetration of external fast time-varying magnetic fields produced by the pulse current in the busbars of the kicker magnet. The screen conductors can be either solid, i.e., as for the LHC MKI [47], or can be applied using a thin coating, i.e., serigraphy [49]. An important advantage of serigraphed screen conductors is the ease of manufacture of the alumina tube, as grooved slots are no longer required. Nevertheless, the material, width and thickness of the conductors must be properly selected. The electrical conductivity of the screen conductors must be rather high to provide a relatively low resistance path for the beam image current. However, at higher frequencies, the eddy currents induced in the screen conductors will modify the magnetic field produced by the kicker magnet: this can unduly degrade the desired field response. In addition, the selected dimensions and electrical conductivity must be compatible with the requirement of achieving low beam coupling impedance. Here we assume that a good electromagnetic shielding is provided when the thickness of the conductor is at least three skin depths, at the lowest frequency of concern. An important concern for the FCC-hh kicker magnet operation is the power deposition in the ferrite yoke, which is significant from the first beam harmonic, i.e., at $40 \mathrm{MHz}$ for $25 \mathrm{~ns}$ bunch spacing. Hence, the thickness of the screen conductors must be selected to ensure effective shielding at this frequency and above. However, the thickness must be compatible with the requirement of good adhesion and quality of the coating. The shielding at lower frequencies, and thus the maximum thickness of the screen conductors, is typically limited by the necessity of good pulse field response.

A continuous coating of a thin metallic layer is frequently applied inside the alumina chambers of kicker magnets [50]. This solution is typically used for lumped inductance kicker magnets, which are generally used when longer field rise times are allowed [11]. In such a case, despite the shielding effect of the induced eddy currents, the required field response can still be achieved. However, for the FCC-hh injection kicker magnets, the specifications for magnetic field rise and fall times and field flat-top quality are much more demanding and hence transmission line kicker magnets are used. In addition, as a result of the field propagation, eddy currents are induced in the longitudinal direction of the beam screen. Hence, capacitive coupling to a conductive cylinder, which is connected to the beam pipe, is introduced at one end of the coating to prevent an eddy current loop through the beam pipe: this excludes the possibility of applying a continuous coating over the entire surface of the alumina tube.

\section{B. Opera-2D model}

The steady-state AC solver of the FEM code Opera-2D was used to compute time-varying electromagnetic fields, including eddy current effects. In FEM, the model is divided into regions. The regions are nonoverlapping areas with specified properties. Finally, a so-called background region is specified for the whole model: Opera-2D fits the background around existing regions. Figure 13 presents the geometry of the problem studied: Only the upper half is modelled due to the top-bottom symmetry. The entire model consists of the following elements: GND busbar (region 1), HV busbar (region 2), ferrite (regions 3-6), screen conductors (regions 7-19), aperture (region 20) and background (region 21). The busbars are modelled as a stainless steel with electrical conductivity of

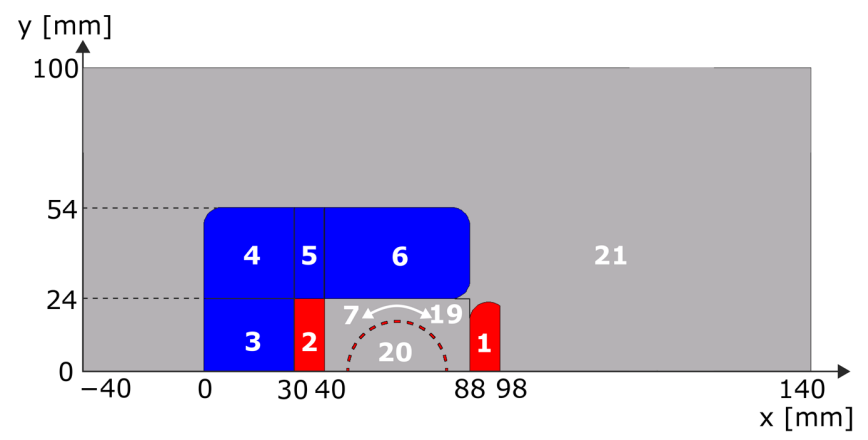

FIG. 13. Geometry of the kicker magnet with a beam screen (Opera-2D model). Due to the top-bottom symmetry only the upper half is modeled. The individual regions are numbered. 
$1.45 \times 10^{6} \mathrm{~S} / \mathrm{m}$ [51]. As discussed in Sec. III, the ferrite dimensions of the FCC-hh injection kicker magnet are chosen such that a central cell operates far from saturation. Hence, the nonlinear BH curve of the ferrite is neglected in the analysis and the real relative permeability of ferrite is modeled as being constant $\left(\mu_{r}=2000\right)$. The width of the busbars is specified to be $10 \mathrm{~mm}$. The geometry of the GND busbar and ferrite have been optimized: both elements have rounded corners to reduce electric field strength and ensure that the specified field homogeneity is achieved in the aperture. In the 2D model, it is assumed that for the infinite length of the structure, the field distribution does not vary along the length and there is no component of field parallel to the longitudinal axis $(z)$. This approximation is reasonable for estimating the central cell inductance, i.e., neglecting end effects.

\section{Computational methods}

In Opera-2D, the predicted stored magnetic energy per unit length $\left(E_{m}\right)$ can be calculated either for the entire model or for specific regions. For a linear magnetic circuit and a given excitation current $(I)$, an equivalent inductance of a central cell, which has a length $l_{\text {cell }}$, can be calculated using the following formula:

$$
L_{\text {cell }}^{\text {Opera-2D }}=\frac{2 E_{m}}{I^{2}} l_{\text {cell }} .
$$

In a similar way, from the predicted power per unit length $\left(P_{m}\right)$, one can calculate the equivalent resistance associated with a central cell $\left(R_{\text {cell }}^{\text {Opera-2D }}\right)$ :

$$
R_{\text {cell }}^{\text {Opera-2D }}=\frac{P_{m}}{I^{2}} l_{\text {cell }}
$$

\section{Frequency dependent inductance}

To study eddy currents induced in the screen conductors and to analyze their effect on the inductance of a central cell of the kicker magnet, as a function of frequency, the simulations were carried out from $0.1 \mathrm{~Hz}$ to $5 \mathrm{MHz}$. The upper frequency limit is determined by the highest frequency of interest in the Fourier transform of the trapezoidal current pulse: $f_{\max } \approx \frac{1}{\pi \tau_{c}}$ [52], where $\tau_{c}$ is the rise time of the current pulse. For the FCC-hh injection kicker magnet, $\tau_{c}=75 \mathrm{~ns}$, while the fall time is expected to be longer [7]. Hence, it follows that $f_{\max } \approx 4.25 \mathrm{MHz}$. To achieve reliable results, the conductors were modelled with a regular mesh with at least 3 mesh-cells for the first skin depth, at the highest frequency of interest [53]. It should be noted, since a 2D simulation code is used, the screen conductors are treated as being straight rather than having a helical shape. In addition, for helical conductors, with an integer number of turns in the aperture, the induced voltage is nominally the same for all screen conductors and

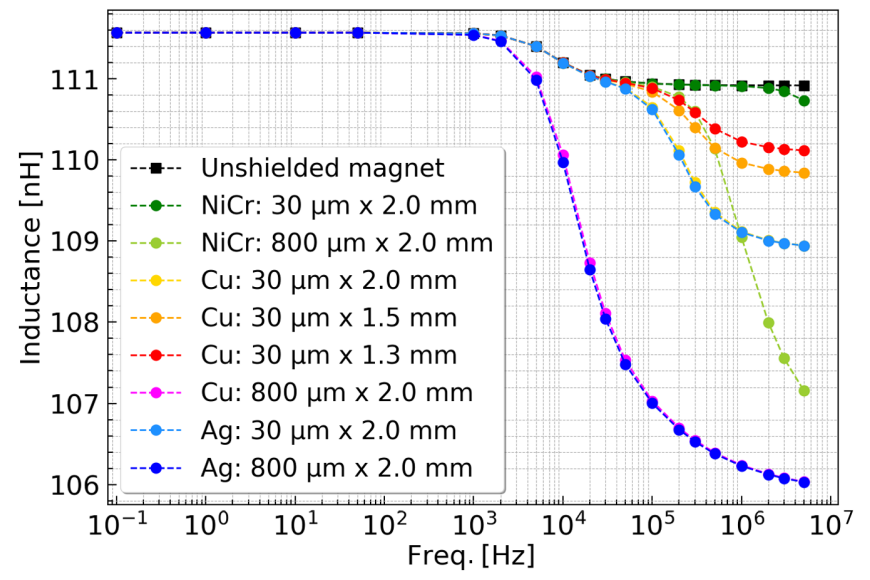

FIG. 14. Inductance of a central cell, excluding internal inductance of busbars, as a function of frequency for the FCC-hh injection kicker magnet with a beam screen, analyzed for different materials, width and thickness of the screen conductors.

hence eddy currents would not flow through one and return through another, even if they are all connected together in the overlap region. To correctly simulate this in Opera-2D, the total induced current in any screen conductor is specified to be 0 A, i.e., the integral of current density over the 2D surface area of any screen conductor is zero.

Several materials for screen conductors were considered, i.e., $\mathrm{NiCr}, \mathrm{Cu}$ and $\mathrm{Ag}$. In addition, we studied different geometries of the screen conductors, by varying their width and thickness. For each configuration, the central cell inductance (see Fig. 14) was evaluated for the entire model excluding busbars, since the internal inductance of the busbars is negligible during the pulse, as the magnetic field is almost completely excluded from the busbars at higher frequencies due to eddy currents [5].

At low frequencies $(\leq 1 \mathrm{kHz})$, the eddy currents induced in the screen conductors are relatively small and, hence, all configurations studied have the same value of low frequency inductance $(111.56 \mathrm{nH})$, which is close to the analytic value for the aperture dimensions [5].

Let us first analyze solid screen conductors with $2 \mathrm{~mm}$ width and $0.8 \mathrm{~mm}$ thickness. The simulations show that above a certain frequency, the cell inductance, as a function of frequency, strongly decreases for higher electrical conductivity. The reduction of inductance for $\mathrm{Cu}$ (pink curve) or Ag (dark blue curve) screen conductors occurs above $1 \mathrm{kHz}$. Whereas, for $\mathrm{NiCr}$ (light green curve) screen conductors, a significant decrease occurs only above $100 \mathrm{kHz}$. For $\mathrm{Cu}$ and $\mathrm{Ag}$ screen conductors the inductance reduces to $106 \mathrm{nH}$ at $5 \mathrm{MHz}$, whereas for $\mathrm{NiCr}$ the inductance reduces to $107.2 \mathrm{nH}$ at $5 \mathrm{MHz}$ : however, the inductance with the $\mathrm{NiCr}$ screen conductors is still decreasing at $5 \mathrm{MHz}$ and, at higher frequencies, would be expected to converge to the same value as for the $\mathrm{Cu}$ and $\mathrm{Ag}$ conductors $(106 \mathrm{nH})$. The inductance characteristics for the $\mathrm{Cu}$ and $\mathrm{Ag}$ conductors are expected to have negative impact 
on the magnetic field response, as the drop occurs significantly below the highest frequency of interest $(\sim 4.25 \mathrm{MHz})$ and has greater amplitude $(\sim 4.4 \%)$, than for the $\mathrm{NiCr}$ conductors $(\sim 3.3 \%)$.

Let us compare the predictions for $\mathrm{Cu}$ (yellow curve, partially hidden under the light blue curve), Ag (light blue curve) and $\mathrm{NiCr}$ (dark green curve) screen conductors, $2 \mathrm{~mm}$ wide and $30 \mu \mathrm{m}$ thick with those with the same materials but for $0.8 \mathrm{~mm}$ thick conductors. As can be observed, the drop in inductance at higher frequencies can be significantly decreased by reducing the thickness of the screen conductors, e.g., for $\mathrm{Ag}$ the inductance at $5 \mathrm{MHz}$ is increased from $106 \mathrm{nH}$ for $0.8 \mathrm{~mm}$ thickness to $109 \mathrm{nH}$ for $30 \mu \mathrm{m}$ thickness. Hence, the reduction in cell inductance is decreased from $4.4 \%$ to $1.7 \%$.

Moreover, considering $2 \mathrm{~mm}$ wide and $30 \mu \mathrm{m}$ thick screen conductors, for $\mathrm{Cu}$ and $\mathrm{Ag}$ the inductance is $109 \mathrm{nH}$ at $5 \mathrm{MHz}$, whereas for $\mathrm{NiCr}$, it is $110.8 \mathrm{nH}$ at $5 \mathrm{MHz}$. The $30 \mu \mathrm{m}$ thick configuration has favorable characteristics for $\mathrm{NiCr}$ : in this case, the frequency dependence of inductance is similar to that of the unshielded kicker magnet (black curve). However, the feasibility of applying $\mathrm{NiCr}$ coating on alumina would require further investigation.

Finally, the predictions for $\mathrm{Cu}$ screen conductors of $30 \mu \mathrm{m}$ thickness and widths of $2 \mathrm{~mm}$ (yellow curve), $1.5 \mathrm{~mm}$ (orange curve) and $1.3 \mathrm{~mm}$ (red curve) show that with reduced width, the inductance drop is smaller at high frequency. For $2 \mathrm{~mm}, 1.5 \mathrm{~mm}$ and $1.3 \mathrm{~mm}$ width, the inductance at $5 \mathrm{MHz}$ is $109 \mathrm{nH}, 109.8 \mathrm{nH}$, and $110.2 \mathrm{nH}$, respectively. These values correspond to a reduction in inductance by $1.9 \mathrm{nH}, 1.1 \mathrm{nH}$, and $0.7 \mathrm{nH}$, respectively, with respect to an unshielded kicker magnet. In particular, similar results are expected for $\mathrm{Ag}$, as both materials have comparable electrical conductivity [5].

For all CST models, the width and thickness of the screen conductors was modeled as per the LHC MKIs $(2.7 \mathrm{~mm} \times 0.8 \mathrm{~mm})$ : hence the thickness of at least 3 skindepths was ensured at $40 \mathrm{MHz}$. Also, we verified that decreasing the width of the screen conductors down to $1 \mathrm{~mm}$ does not influence the beam coupling impedance predictions. Hence, the $30 \mu \mathrm{m}$ thick $\mathrm{Cu}$ or $\mathrm{Ag}$ screen conductors are expected to provide low broadband beam coupling impedance from $40 \mathrm{MHz}$, as their thickness is equal to 3 skin depths at this frequency [5]. Moreover, the screen conductors with this thickness should sustain the beam image current and eddy current heating, as the $\mathrm{Ti}$ coating in the LHC dump kicker is only $2 \mu \mathrm{m}$ thick [50] and has $\sim 10$ times higher resistivity, but no issues have occurred. However, this aspect requires further studies as the FCC-hh injection system must operate at $10 \mathrm{~Hz}$ burst rate [1].

\section{E. PSpice equivalent circuit}

In Opera-2D, the capacitance of each cell to ground of the transmission line kicker magnet cannot presently be modeled. Hence, neither the propagation delay time of the pulse through the kicker magnet nor the cutoff frequency of a cell is taken into account in Opera-2D. Similarly, end effects are neglected. To analyze the field response of the kicker magnet with a beam screen, our approach involves modelling, using PSpice Optimizer, the equivalent circuit of the frequency dependent inductance of each cell in the kicker magnet. In this method, an equivalent circuit of the frequency dependent inductance and resistance of a central cell is used [54]. The basic "building block" of the equivalent circuit consists of a parallel connected resistor and inductor whose values are frequency independent. To simulate a wide frequency range, several of these blocks are connected in series. The PSpice Optimizer is used to determine the resistance and inductance values, which minimizes the RMS error between the Opera-2D predicted frequency dependent inductance and resistance and those of the equivalent circuit.

For a beam screen composed of $24 \mathrm{Cu}$ screen conductors with $30 \mu \mathrm{m}$ thickness and $1.3 \mathrm{~mm}$ width, the frequency dependent inductance and resistance have been quantified using Opera-2D. The results derived from the Opera-2D predictions, using Eqs. (8) and (9), are indicated by dashed lines in Fig. 15. The aforementioned characteristics were fitted to an equivalent circuit using PSpice together with the PSpice Optimizer [5]. The obtained fits are depicted by solid lines in Fig. 15.

The equivalent circuit utilized to simulate the frequency dependent inductance and resistance is shown in Fig. 16. It consists of four building blocks to provide an accurate fit ( $\sim 0.01 \%$ maximum error for the inductance) within the frequency range from $1 \mathrm{kHz}$ to $5 \mathrm{MHz}$. At low frequency, the current flows through the parallel inductors, bypassing all the resistors. The sum of the parallel inductors and the inductor in series must be equal to the inductance of a central cell at low frequency, i.e., $111.56 \mathrm{nH}$ (see red dashed line in Fig. 15). As the frequency increases, the

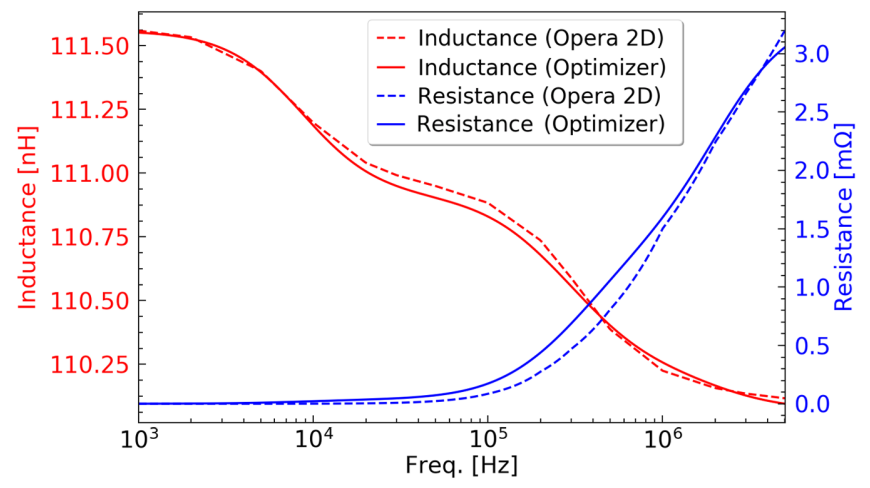

FIG. 15. Frequency dependent inductance and resistance of the FCC-hh injection kicker magnet with a beam screen composed of $24 \mathrm{Cu}$ screen conductors $(30 \mu \mathrm{m} \times 1.3 \mathrm{~mm})$. Opera-2D data is compared with the fit of the equivalent circuit obtained using PSpice and the PSpice Optimizer. 


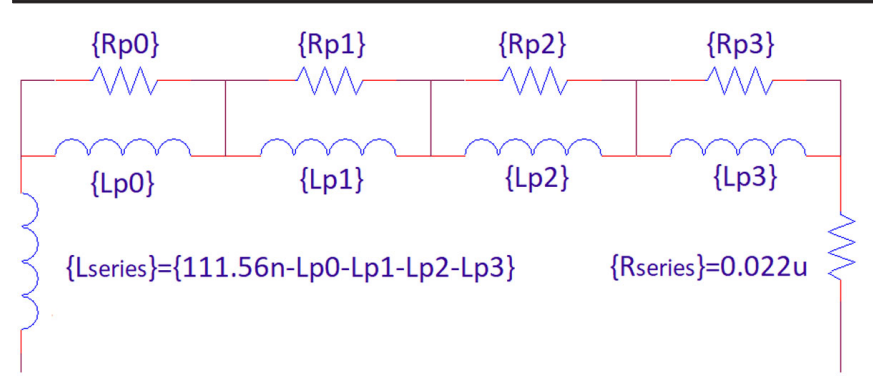

FIG. 16. Equivalent circuit of the frequency dependent inductance per cell of the kicker magnet.

impedance of the inductors increases: at very high frequencies the impedance of the inductors will be such that the current flows predominantly through the parallel resistors, bypassing the parallel inductors. Thus, the value of the series inductor (Lseries) must be equal to the high frequency inductance of the cell. In addition, the sum of the resistors should be equal to the resistance of a central cell at high frequency, i.e., $3.2 \mathrm{~m} \Omega$ at $5 \mathrm{MHz}$ (see blue dashed line in Fig. 15). Hence, the circuit exhibits the following behavior: as the frequency increases, the effective inductance decreases and the effective resistance increases. The value of resistance in series with the building blocks is relatively small [54], and is modelled to avoid "inductive loops" in the PSpice model. For the optimization of the values, we provided an initial guess for the component parameters. The initial guesses are based on predictions from trial simulations where all the values were changed randomly within a specified range: this was to try and identify a starting point near to a global minimum. The final component values were found using the PSpice Optimizer to minimize the RMS error between the Opera-2D characteristics and those predicted from the equivalent circuit. During the optimization, the fitting of the inductance characteristic is deliberately weighted more heavily than the fitting of the resistance curve, since the inductance is most relevant for calculating the magnetic field in the aperture [5]. The maximum error of the equivalent inductance and resistance is $0.01 \%$ and $7.8 \%$, respectively. The $0.01 \%$ maximum error in the fitted inductance is considered acceptable, as it is considerably less than the permissible field error $( \pm 0.5 \%)$.

\section{F. Benchmark simulations of field-rise time and field flat-top quality}

To examine the impact of the proposed beam screen upon the field response of the kicker magnet, we performed Cadence PSpice simulations. In the equivalent circuit of the transmission line kicker magnet shown in Fig. 1, the frequency independent inductance of each cell was replaced by the equivalent circuit of the frequency dependent inductance shown in Fig. 16. For a 20-cell FCC-hh injection kicker magnet, a physical length of a central cell is $l_{\text {cell }}=0.088 \mathrm{~m}$, as the total aperture length of one module is $1.765 \mathrm{~m}$. The effective length of an end cell $\left(l_{\text {end }}\right)$, in comparison with the physical length of a central cell $\left(l_{\text {cell }}\right)$, can be estimated using the following formula [11]:

$$
l_{\text {end }}=l_{\text {cell }}+0.25 V_{\text {ap }},
$$

where $V_{\text {ap }}$ is the aperture dimension in a plane perpendicular to the beam motion. The inductance of an end cell can be calculated as follows:

$$
L_{\text {end }}=\frac{l_{\text {end }}}{l_{\text {cell }}} L_{\text {cell }} \text {. }
$$

For the selected aperture dimensions ( $V_{\text {ap }}=48 \mathrm{~mm}$ ), by applying Eq. (10), $l_{\text {end }}=0.1 \mathrm{~m}$ : this is $13.6 \%$ greater than a central cell. Hence, to account for end effects, a series inductance of $15 \mathrm{nH}\left(L_{\text {end }}-L_{\text {cell }}\right)$ has been added at both ends of the kicker magnet, directly in series with the equivalent circuit of the frequency dependent inductance of the end cells. If the series resistance, shown in Fig. 16, is sufficiently small, the magnetic flux pulse in the kicker magnet can be calculated from:

$$
\Phi=\int\left(V_{\text {in }}-V_{\text {out }}\right) \cdot d t
$$

$V_{\text {in }}$ and $V_{\text {out }}$ are defined in Fig. 1.

Studies show that for a 20-cell kicker magnet with a proposed beam screen, the specifications for both the magnetic field rise time and field flat-top quality are obtained with the following parameters: $R_{\text {cell }}=25.5 \Omega$, $C_{\text {cell }}=2.84 \mathrm{nF}, C_{\text {out }}=2.33 \mathrm{nF}$ and $C_{\text {in }}=1.2 \mathrm{nF}$ [5]. Figure 17 shows the waveform of a 20-cell FCC-hh injection kicker magnet with a proposed beam screen (red curve), in comparison with that of the unshielded kicker magnet (green curve). Both waveforms cross the $0.5 \%$ threshold of the normalized magnetic flux at an elapsed time of $\sim 15 \mathrm{~ns}$. To achieve the required magnetic

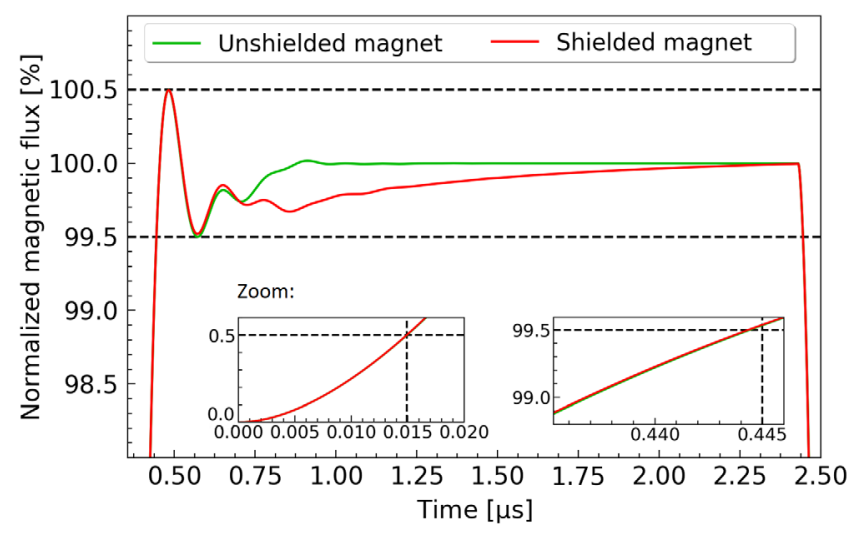

FIG. 17. Comparison of the predicted normalized magnetic flux for a 20-cell FCC injection kicker magnet with the beam screen (red curve) and without the beam screen (green curve). 
field rise time, each waveform must cross $99.5 \%$ of the normalized magnetic flux by an elapsed time of $445 \mathrm{~ns}$ $(430 \mathrm{~ns}+15 \mathrm{~ns})$. As can be seen, in both cases this requirement is met (see zoom in Fig. 17). In addition, we can observe that the beam screen causes a time dependent reduction in the normalized magnetic field with respect to the unshielded kicker magnet. However, the amplitude of the first overshoot and first undershoot is very similar to that of the unshielded kicker magnet. Although this flat-top field is still within specification, and is thus acceptable, it may be possible to modulate the output of the IA to compensate for the overshoot and undershoot [55], as well as the influence of the beam screen-this option would require further study. To conclude, the proposed beam screen is expected to give a field response which is still within specification, but might be further improved by suitable modulation of the IA.

\section{BENCHMARK MEASUREMENTS}

This section presents benchmark measurements of the prototype helical beam screen that has been manufactured at CERN. The first prototype was not optimized either for the FCC-hh nor for the LHC MKI injection kicker magnets in terms of beam coupling impedance and HV operation. The aim was to validate the performance of the helical beam screen with the new features, with limited resources available, by performing benchmark longitudinal impedance measurements. Hence, a prototype helical beam screen with the proposed features, i.e., symmetric vacuum gap, ungraded screen conductors and shorter overlap length $\left(L_{\text {overlap }}=44 \mathrm{~mm}\right)$, which are described in Sec. VI, was installed in a spare LHC MKI.

\section{A. Prototype}

The first prototype of the helical beam screen consists of 21 screen conductors, applied using vacuum compatible $\mathrm{Ag}$ paint $\left(\sigma=3.78 \times 10^{7} \mathrm{~S} / \mathrm{m}\right)$ from Heraeus [56]. To produce the helical beam screen, the inner surface of the alumina tube was coated uniformly with Ag paint. After sintering, a photoresist was applied over the entire surface of the coating. Next, a patterned mask with the helixes made of $\mathrm{Cu}$ paint, applied to a clear sheet, was inserted in the alumina tube and a specially built $3 \mathrm{~m}$-long light tube, designed to give a UVA spectrum over 360 degrees, was mounted inside the tube. In this process, the mask is used to block the light, so that the UVA effects the photoresist where there is no $\mathrm{Cu}$, hence it can be later removed by etching. The applied mask must be in good contact with the substrate to prevent the UVA effecting the photoresist under the $\mathrm{Cu}$. Finally, an etching agent was used to remove Ag in areas where the mask was not placed: this is a difficult step, i.e., if the photoresist is damaged, where it should be protected, this can result in the etching agent giving breaks in the Ag paint. Another problem is a difficulty to obtain a uniform thickness Ag layer after sintering - small voids can be visible after this process. Thus, in the future, it is proposed that more than one layer of uniform $\mathrm{Ag}$ is applied to the inside of the alumina tube.

In the prototype beam screen, each screen conductor has a width of $\sim 3 \mathrm{~mm}$, but the thickness is not precisely defined. The prototype helical beam screen extends over $\sim 4.5$ turns along the aperture of the LHC MKI. The length of the alumina tube is $3 \mathrm{~m}$ : this is $7 \mathrm{~mm}$ less than the total length of the original alumina tube installed in the LHC MKI. For this reason, special components were designed and produced to extend the prototype tube at the grounded end, so that the helical beam screen could be installed in the spare LHC MKI. A photograph of the prototype helical beam screen is shown in Fig. 18.

Several tests were performed to assess the quality of the $\mathrm{Ag}$ helixes. The voltage drop due to applying a DC current from end-to-end of each helix was measured. The measurements showed that 12 stripes are broken: such a problem could arise during the production process. Damage of the photoresist in the areas where it should be protected can result in the etching agent giving breaks in the Ag paint. For this reason, the manufacturing method should be improved to give more reliable results. In addition, the capacitance from end-to-end of the helixes with the breaks was measured [5]. Significant differences in the measured end-to-end capacitance, of broken helixes, indicated that overall damage and possibly the number of breaks varies between stripes. The average thickness of a helical screen conductor was calculated from measured end-to-end resistance: it should be noted that this is $\sim 12 \mu \mathrm{m}$, in comparison to $39 \mu \mathrm{m}$ required to give 3 skin depths at $40 \mathrm{MHz}$ [5].

\section{B. CST model}

The prototype helical beam screen has also been modeled in CST as being installed in an MKI. The simulations

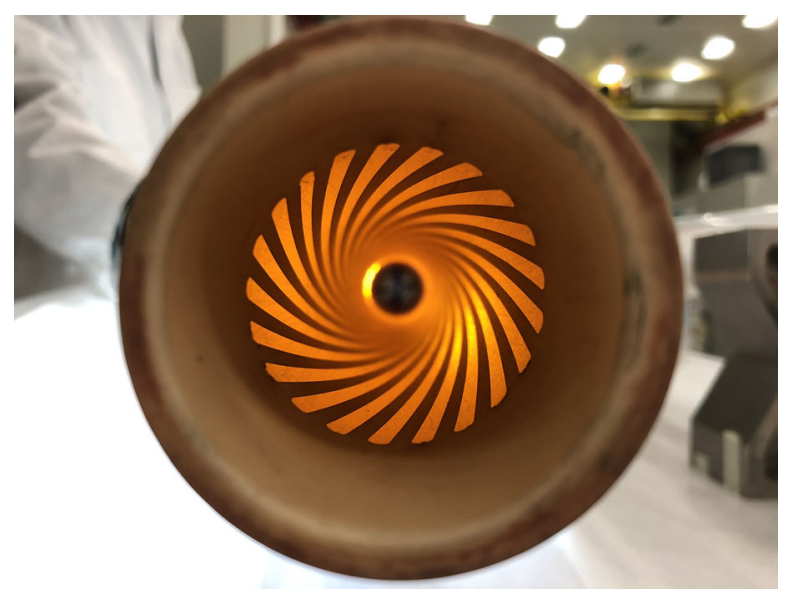

FIG. 18. Photograph of the prototype helical beam screen with 21 screen conductors applied using vacuum compatible Ag paint and 4.5 turns along the length of the alumina tube. 


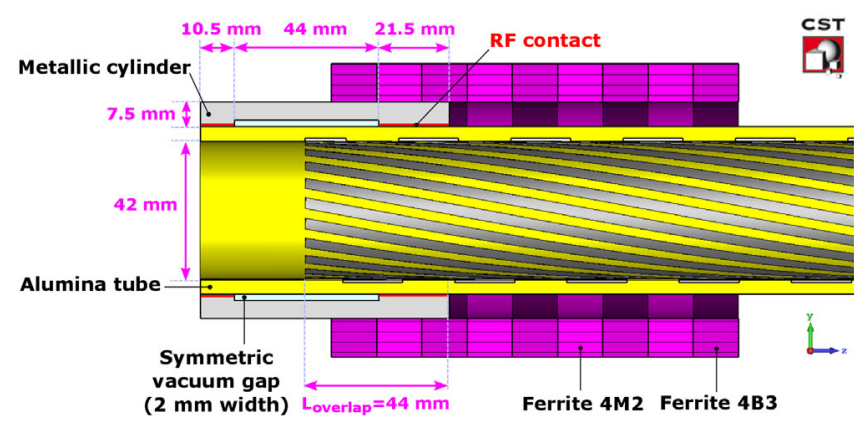

FIG. 19. CST model of a prototype helical beam screen installed in the LHC MKI.

have been performed using the simplified model (i.e., full length beam screen, with ferrite rings at both ends, but excluding the magnet), due to limited computational resources. A zoom of the upstream end of the beam screen is shown in Fig. 19. The thickness of the metallic cylinder has been adjusted to fit with the original Ferroxcube 4M2 and 4B3 ferrite rings, which are alternately arranged at both ends of the LHC MKI alumina tube [28]. Note, in the CST model, it is assumed that the screen conductors have a thickness of at least 3 skin depths at $40 \mathrm{MHz}$, which is not the case with the prototype beam screen due to its $\sim 12 \mu \mathrm{m}$ thickness. A $\sim 12 \mu \mathrm{m}$ thickness corresponds to 3 skindepths at a frequency of $\sim 415 \mathrm{MHz}$.

\section{Experimental setup}

The test bench, of the measurement setup for impedance measurements, consists of an LHC MKI with a helical beam screen. A single $0.5 \mathrm{~mm}$ diameter $\mathrm{Cu}$ wire with a $\sim 2.1 \mu \mathrm{m} \mathrm{Ag}$ coating has been stretched along the center of the aperture of the device under test (DUT). In addition, we used a vector network analyzer (VNA), calibration kit (85032B/E) and rf measurement cables (ST18A/11N468/ 11N468) from Huber + Suhner [57]. For a circular beam pipe and wire the characteristic impedance of the coaxial line is given by $Z_{L}=60 \ln \frac{D}{d}$, where $D$ is the inside diameter of the alumina tube $(42 \mathrm{~mm})$ and $d$ is the wire diameter [3]. In the classical method, the coaxial line impedance $\left(Z_{L}=265 \Omega\right)$ was reasonably well matched to that of the VNA $\left(Z_{0}=50 \Omega\right)$ by using "noninductive," carbon matching resistors $(220 \Omega)$. The reflection coefficient, due to the mismatch of the coaxial line impedance $(265 \Omega$ ) and the $270 \Omega$ resulting from the sum of the VNA impedance with the matching resistor value is small $\left(\frac{(270-265)}{(270+265)}=0.009\right)$. The matching resistors were housed in a short (40 mm long) metallic, nonmagnetic, box (Sucobox). Ideally, however, the Sucoboxes used in this method should be shorter to minimize the parasitic inductance and capacitance in order to improve the accuracy at higher frequencies. For the resonant method [3], Sucoboxes with capacitive coupling have been used. The length of the
LHC MKI between the Sucoboxes is $L_{\text {DUT }}=3.5 \mathrm{~m}$. In addition, in the practical realization, an important aspect is the Ag metallization, indicated by the red color in Fig. 19, that has to be applied to part of the outside diameter of the alumina tube to ensure a good, homogeneous, rf contact between the metallic cylinder and the alumina tube.

\section{Results}

Figure 20 presents the experimental results obtained using classical (grey curve) and resonant (red points) single wire methods [3]. CST predictions (blue curve) are also shown. The resonant coaxial wire method provides good accuracy for the measurements but relatively poor frequency resolution $(\Delta f)$ : frequency data points are spaced such that $\Delta f \simeq(n \cdot c) /\left(2 \cdot L_{\text {DUT }}\right)$ [3,58]: where, $n$ is an integer and $c$ is the speed of light. For the measurements reported here, the frequency resolution is $\sim 40 \mathrm{MHz}$. The classical coaxial wire technique gives an improved frequency resolution. However, any residual mismatch in the characteristic impedance, for example due to nonzero inductance of matching resistors, between the measurement network and the DUT, will result in reflections in the system which limit the achievable accuracy [58]. Nevertheless, the classical coaxial wire technique allows one to see high peaks, that stand out from the background noise, which may fall between measurement points provided by the resonant method: no such peaks are visible here. Hence, the CST predictions are compared with the resonant method measurements: reasonable agreement has been achieved, particularly up to $\sim 1.2 \mathrm{GHz}$.

From simulations, the fundamental mode is expected at $\sim 1230 \mathrm{MHz}$. Indeed, the measurements show that the impedance peak due to the overlap at the upstream end of the beam screen is pronounced between $1200 \mathrm{MHz}$ and $1500 \mathrm{MHz}$. In addition, the results of the classical wire method indicate small resonances between $20 \mathrm{MHz}$ and $150 \mathrm{MHz}$, which are not present in the impedance spectrum

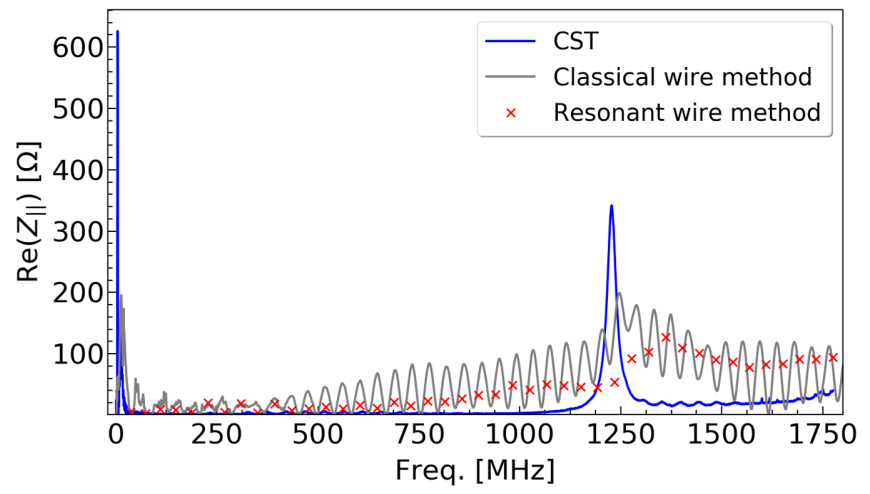

FIG. 20. Results of the real longitudinal impedance measurements performed on the LHC MKI with a prototype helical beam screen using classical and resonant single wire methods. The experimental data is compared with the CST predictions. 
of the LHC MKI with the conventional beam screen [5]. However, an increase of the real part of the longitudinal impedance in the low frequency range (except up to $10 \mathrm{MHz}$ ) can be attributed to the defects of the helix, i.e., thickness of only $12 \mu \mathrm{m}$ and breaks in the silver of 12 screen conductors, in unknown location(s) along their length. Since the capacitive impedance of the break is inversely proportional to frequency, this effect is dominant at low frequencies. To conclude, a prototype helical beam screen with improved coating quality and larger coating thickness, i.e., $39 \mu \mathrm{m}$ to provide 3 skin depths at $40 \mathrm{MHz}$, is proposed as a subject for future impedance measurements.

\section{CONCLUSIONS}

In this paper, we have presented a detailed analysis of the beam screen for the FCC-hh injection kicker magnets. These studies are particularly challenging due to the large size and complexity of the kicker magnet design from the electromagnetic point of view, and due to many technological constraints inherent to the functional specifications of the kicker magnet. First, we optimized the FCC-hh injection kicker system to reduce its length from $130 \mathrm{~m}$ to $40 \mathrm{~m}$. Subsequently we developed a conceptual design of a single FCC-hh injection kicker magnet module. Studies show that at least a 20-cell transmission line kicker magnet must be used to satisfy the magnetic field rise time and field flat-top quality specification. Next, we proposed an innovative design - the helical beam screen, to meet the demanding requirements for fast pulsed kicker magnets of the FCC-hh. The analysis of the helical beam screen has been performed from a theoretical, numerical and experimental point of view, and the results obtained are consistent. The main advantage of the helical beam screen is the possibility to achieve significantly improved HV performance, together with adequately low beam coupling impedance, in comparison with the conventional beam screen design used to date. In particular, we showed that for an integer number of turns in the aperture, the induced voltage on every helical screen conductor is nominally identical and this is approximately one-half of the worst case voltage induced on a straight screen conductor. Hence, with a helical screen design, the surface flashovers of the beam screen are expected to be eliminated.

The longitudinal impedance modes of the helical beam screen are determined by the length of the screen conductors in the overlap region. To improve the design, new features were proposed, i.e., an overlap length reduced to $44 \mathrm{~mm}$, a symmetric vacuum gap and ungraded lengths of screen conductors. Also, we investigated an alternative design of the helical beam screen with screen conductors connected together, outside of the magnet aperture. In the future, this concept could offer the possibility to further reduce beam coupling impedance once injection is completed.
Another important advantage of the helical beam screen is the possibility to control the transverse dipolar impedance, i.e., by adjusting the number of turns, which allows to tune the resonant frequencies of the dipolar modes. For instance, with the given transverse impedance budget, only the helical design can be used in an FCC-hh kicker system: for multiple magnets with the conventional beam screen, it is not possible to tune resonant frequencies without a destructive impact on the longitudinal impedance. Nevertheless, the concept of tuning of the resonant frequencies of the transverse dipolar modes requires further investigation together with benchmarking of the beam coupling impedance for helical designs with different number of turns: to provide a beam coupling impedance estimate each beam screen would have to be analyzed separately.

In addition, we investigated an optimal material, width and thickness of the screen conductors. The baseline design will consist of $24 \mathrm{Cu}$ or $\mathrm{Ag}$ screen conductors, $30 \mu \mathrm{m}$ thick and $1.3 \mathrm{~mm}$ wide. Simulations show that the proposed beam screen will provide a field response which is within specifications. Finally, we measured the longitudinal impedance of the prototype helical beam screen, consisting of 21 screen conductors, applied using a vacuum compatible Ag paint, which has been installed in the LHC MKI. The benchmark measurements confirmed that the longitudinal impedance is determined by the capacitive coupling and the first peak is pronounced between $1200 \mathrm{MHz}$ to $1500 \mathrm{MHz}$. Small resonances below $150 \mathrm{MHz}$ are attributed both, to the defects of the helical conductors and an average thickness of only $12 \mu \mathrm{m}$, which corresponds to only one skin depth at $40 \mathrm{MHz}$. Therefore, future developments are required to suitably apply the helix to an alumina tube. To guarantee effective shielding, an improved coating quality, without breaks and with adequate thickness, should be studied. To summarize, the studies presented give a complete outline of the design, optimization and measurements of a new beam screen for fast, transmission line type, kicker magnets. The achieved results provide a solid base for future studies toward the implementation of a helical beam screen in kicker magnets of new or existing machines.

\section{ACKNOWLEDGMENTS}

The authors would like to thank L. Ducimetière and $\mathrm{W}$. Weterings for help with the design and modification of parts to permit installation of the prototype helical beam screen in the LHC MKI. Also, we would like to thank G. Bellotto for help with preparation of the measurement setup. The authors are grateful to F. Caspers for fruitful discussions. We acknowledge S. Arsenyev for providing expertise regarding the FCC-hh transverse impedance model. In addition, we would like to thank E. Shaposhnikova, A. Lasheen and I. Karpov for useful discussions about longitudinal beam dynamics. Special thanks to M. Alandes Pradillo and P. Llopis Sanmillan for good support with the computing infrastructure. 
[1] A. Abada et al., FCC-hh: The Hadron Collider. Future Circular Collider Conceptual Design Report Volume 3, Eur. Phys. J. Spec. Top. 228, 755 (2019).

[2] L. Ducimetière, N. Garrel, M. J. Barnes, and G. D. Wait, The LHC injection kicker magnet, in Proceedings of Particle Accelerator Conference (PAC'03), Portland, Oregon, USA, 12-16 May, 2003 (IEEE Operations Center, Piscataway, USA, 2003), pp. 1162-1164.

[3] T. Kroyer, F. Caspers, and E. Gaxiola, Longitudinal and transverse wire measurements for the evaluation of impedance reduction measures on the MKE extraction kickers, CERN Technical Report No. CERN-AB-Note-2007-028, 2007.

[4] A. Chmielińska, M. J. Barnes, W. Bartmann, F. Burkart, and B. Goddard, Preliminary estimate of beam induced power deposition in a FCC-hh injection kicker magnet, in Proceedings of the 8th International Particle Accelerator Conference (IPAC'17), Copenhagen, Denmark, 14-18 May, 2017, International Particle Accelerator Conference No. 8 (JACoW, Geneva, Switzerland, 2017), pp. 3475-3478.

[5] A. Chmielińska, Optimization of the beam screen for the FCC injection kicker magnets, Ph.D. thesis, École Polytechnique Fédérale de Lausanne (2019), https://infoscience .epfl.ch/record/273161/files/EPFL_TH8079.pdf.

[6] CERN Impedance Website, FCC-hh Impedance, https:// impedance.web.cern.ch/impedance/fcchh/impedances .html.

[7] D. Woog, M. J. Barnes, L. Ducimetière, J. Holma, and T. Kramer, Design of an Inductive Adder for the FCC injection kicker pulse generator, J. Phys. Conf. Ser. 874, 012096-1 (2017).

[8] H. Day, Measurements and simulations of impedance reduction techniques in particle accelerators, Ph.D. thesis, University of Manchester, 2013, https://cds.cern.ch/record/ 1564644/files/CERN-THESIS-2013-083.pdf.

[9] T. Kramer, M. J. Barnes, W. Bartmann, F. Burkart, L. Ducimetière, B. Goddard, V. Senaj, T. Stadlbauer, D. Woog, and D. Barna, Considerations for the injection and extraction kicker systems of a $100 \mathrm{TeV}$ centre-of-mass FCC-hh collider, in Proceedings of the 7th International Particle Accelerator Conference (IPAC'16), Busan, Korea, 8-13 May, 2016, International Particle Accelerator Conference No. 7 (JACoW, Geneva, Switzerland, 2016), pp. 3901-3904.

[10] E. Renner, Machine protection of the Future Circular Hadron Collider FCC-hh: Injection and extraction, Master's thesis, Vienna University of Technology, 2018, http:// cds.cern.ch/record/2648770/files/CERN-THESIS-2018261.pdf.

[11] M. J. Barnes, Kicker systems, in Proceedings of CASCERN Accelerator School: Beam Injection, Extraction and Transfer (2017), pp. 229-283, https://doi.org/10.23730/ CYRSP-2018-005.229.

[12] M. J. Barnes, A. Adraktas, G. Bregliozzi, B. Goddard, L. Ducimetière, B. Salvant, J. Sestak, L. V. Cid, V. Vlachodimitropoulos, W. Weterings, and C. Y. Vallgren, Operational experience of the upgraded LHC injection kicker magnet during run 2 and future planes, J. Phys. Conf. Ser. 874, 012101-1 (2017).
[13] M. J. Barnes, W. Bartmann, F. Burkart, L. Ducimetière, B. Goddard, T. Kramer, V. Senaj, T. Stadlbauer, D. Woog, D. Barna, L. M. Redondo, and A. Kandratsyeu, Future circular collider injection and extraction kicker topologies and solid state generators, Phys. Rev. Accel. Beams 22, 071001-1 (2019).

[14] L. M. Redondo, A. Kandratsyeu, and M. J. Barnes, Marx generator prototype for kicker magnets based on $\mathrm{SiC}$ MOSFETs, IEEE Trans. Plasma Sci. 46, 3334 (2018).

[15] M. J. Barnes, L. Ducimetière, J. Holma, T. Kramer, and A. Fowler, Inductive adders for replacing thyratron based modulators at CERN, in Proceedings of the 17th European Conference on Power Electronics and Applications (EPE'15 ECCE-Europe), Geneva, Switzerland, 8-10 September, 2015 (IEEE, New York, 2015), pp. 73091607309170, https://doi.org/10.1109/EPE.2015.7309160.

[16] L. Vos, Longitudinal impedance from ferrite, CERN Technical Report No. CERN-SL-2000-010 AP, 2000.

[17] National Magnetics Group, Inc., CMD5005, General Purpose, High Frequency Ni-Zn Ferrite, (2019), http:// magneticsgroup.com/wp-content/uploads/2019/09/ CMD5005-ISO-WEB-DATA.pdf.

[18] M. J. Barnes, V. Mertens, N. Garrel, B. Goddard, W. Weterings, and L. Ducimetière, Analysis of ferrite heating of the LHC injection kickers and proposals for future reduction of temperature, in Proceedings of the $3 \mathrm{rd}$ International Particle Accelerator Conference (IPAC'12), New Orleans, Louisiana, USA, 20-25 May, 2012, International Particle Accelerator Conference No. 3 (JACoW, Geneva, Switzerland, 2012), pp. 2038-2040.

[19] D. Woog, A. F. Colomo, T. Kramer, and M. J. Barnes, First prototype inductive adder for the FCC injection, in Proceedings of 9th International Particle Accelerator Conference (IPAC18), Vancouver, Canada, 29 April-4 May, 2018, International Particle Accelerator Conference No. 9 (JACoW, Geneva, Switzerland, 2018), pp. 25532556.

[20] Allegro PSpice System Designer-Cadence, https://www .cadence.com/ (2019).

[21] CST Studio Suite, http://www.cst.com/ (2019).

[22] J. Cao (private communication).

[23] A. Chmielińska, B. Popovic, M. J. Barnes, F. Caspers, and C. Vollinger, Measurements of electromagnetic properties of ferrites as a function of frequency and temperature, J. Phys. Conf. Ser. 1067, 082018-1 (2018).

[24] V. Vlachodimitropoulos, M. J. Barnes, and A. Chmielińska, Preliminary results from validation measurements of the longitudinal power deposition model for the LHC injection kicker magnet, in Proceedings of the 9th International Particle Accelerator Conference (IPAC'18), Vancouver, Canada, 29 April-4 May, International Particle Accelerator Conference No. 9 (JACoW, Geneva, Switzerland, 2018), pp. 2636-2639.

[25] V. Vlachodimitropoulos, M. J. Barnes, L. Ducimetière, L. V. Cid, and W. Weterings, Longitudinal impedance analysis of an upgraded LHC injection kicker magnet, in Proceedings of the 9th International Particle Accelerator Conference (IPAC'18), Vancouver, Canada, 29 April-4 May, International Particle Accelerator 
Conference No. 9 (JACoW, Geneva, Switzerland, 2018), pp. 2628-2631.

[26] Ferroxcube, 4B3 Material Specification, https://www .ferroxcube.com/upload/media/product/file/MDS/4b3.pdf (2008).

[27] Ferroxcube, 4M2 Material Specification, https://www. ferroxcube.com/upload/media/product/file/MDS/4m2.pdf (2008).

[28] M. J. Barnes, F. Caspers, H. Day, M. Garlaschè, W. Weterings, M. Taborelli, L. Ducimetière, S. Calatroni, V. G. Namora, V. Mertens, and J. Uythoven, Beam induced ferrite heating of the LHC injection kickers and proposals for improved cooling, in Proceedings of the 4th International Particle Accelerator Conference (IPAC'13), Shanghai, China, 12-17 May, 2013, International Particle Accelerator Conference No. 4 (JACoW, Geneva, Switzerland, 2013), pp. 732-734.

[29] National Magnetics Group, Inc., CMD10: High Flux Density, High Frequency Ni-Zn Ferrite, https://www .magneticsgroup.com/wp-content/uploads/2019/09/ CMD10-ISO-WEB-DATA.pdf (2019).

[30] M. J. Barnes, P. Adraktas, F. Caspers, B. Teissandier, M. Taborelli, L. Ducimetière, R. Noulibos, S. Calatroni, V. G. Namora, V. Mertens, and J. Uythoven, Reduction of surface flashover of the beam screen of the LHC injection kickers, in Proceedings of the 4th International Particle Accelerator Conference (IPAC'13), Shanghai, China, 12-17 May, 2013, International Particle Accelerator Conference No. 4 (JACoW, Geneva, Switzerland, 2013), pp. 735-738.

[31] M. J. Barnes, F. Caspers, L. Ducimetière, N. Garrel, and T. Kroyer, An improved beam screen for the LHC injection kickers, in Proceedings of Particle Accelerator Conference 2007 (PAC'07), Albuquerque, New Mexico, USA, 25-29 June, 2007 (IEEE, Piscataway, USA, 2007), pp. 1162-1164.

[32] A. Adraktas, H. Day, L. Ducimetière, and M. J. Barnes, High voltage performance of surface coatings on alumina insulators, in Proceedings of the 7th International Particle Accelerator Conference (IPAC'16), Busan, Korea, 8-13 May, 2016, International Particle Accelerator Conference No. 7 (JACoW, Geneva, Switzerland, 2016), pp. 3603-3606.

[33] M. J. Barnes, L. V. Cid, T. Kramer, G. Bregliozzi, Y. C. Vallgren, A. Chmielińska, C. Bracco, B. Goddard, H. Neupert, L. Ducimetière, and V. Vlachodimitropoulos, An upgraded LHC injection kicker magnet, in Proceedings of the 9th International Particle Accelerator Conference (IPAC'18), Vancouver, Canada, 29 April-4 May, International Particle Accelerator Conference No. 9 (JACoW, Geneva, Switzerland, 2018), pp. 2632-2635.

[34] H. Day, L. V. Cid, L. Ducimetière, M. J. Barnes, and W. Weterings, Current and future beam thermal behaviour of the LHC injection kicker magnet, in Proceedings of the 7th International Particle Accelerator Conference (IPAC'16), Busan, Korea, 8-13 May, 2016, International Particle Accelerator Conference No. 7 (JACoW, Geneva, Switzerland, 2016), pp. 3615-3618.

[35] V. Vlachodimitropoulos, M. J. Barnes, L Ducimetière, L. V. Cid, and W. Weterings, Study of an improved beam screen design for the LHC injection kicker magnet for HL-LHC, in Proceedings of the 8th International Particle Accelerator Conference (IPAC'17), Copenhagen, Denmark, 14-18 May, 2017, International Particle Accelerator Conference No. 8 (JACoW, Geneva, Switzerland, 2017), pp. 3471-3474.

[36] M. J. Barnes, C. Bracco, G. Bregliozzi, A. Chmielinska, L. Ducimetière, B. Goddard, G. Iadarola, T. Kramer, L. V. Cid, V. Vlachodimitropoulos, and W. Weterings, Operational experience of a prototype LHC injection kicker magnet with a low SEY coating and redistributed power deposition, J. Phys. Conf. Ser. 1350, 012145 (2019).

[37] L. V. Cid, A. Abnades, M. J. Barnes, F. Mostchmann, V. Vlachodimitropoulos, and W. Weterings, Conception and design of a cooling system for the LHC injection kicker magnets, Nucl. Instrum. Methods Phys. Res., Sect. A 916, 296 (2019).

[38] CST Studio Suite, CST Particle Studio Wakefield solver, https://www.cst.com/products/cstps/solvers/wake fieldsolver (2019).

[39] G. Rumolo, Beam Instabilities, in Proceedings of CASCERN Accelerator School: Advanced Accelerator Physics Course (2013), pp. 199-220, https://doi.org/10.5170/ CERN-2014-009.199.

[40] E. Shaposhnikova, Longitudinal stability of the LHC beam in the SPS, CERN Technical Report No. SL-Note-2001031-HRF, 2001).

[41] I. Karpov and E. Shaposhnikova, Longitudinal coupledbunch instability evaluation for fcc-hh, in Proceedings of 10th International Particle Accelerator Conference (IPAC19), Melbourne, Australia, 19-24 May, 2019, International Particle Accelerator Conference No. 10 (JACoW, Geneva, Switzerland, 2019), pp. 297-300.

[42] CST Studio Suite, CST Microwave Studio Eigenmode solver, https://perso.telecom-paristech.fr/begaud/intra/MWS_ Tutorials.pdf (2006).

[43] CST Studio Suite, HF design and analysis, https://perso .telecom-paristech.fr/begaud/intra/CTS_MWS_ AdvancedTopics.pdf (2003).

[44] S. Arsenyev and B. Salvant, A method for computing driving and detuning beam coupling impedances of an asymmetric cavity using eigenmode simulations, arXiv: 1904.04680.

[45] B. W. Zotter and S. A. Kheifets, Impedances and Wakes in High-Energy Particle Accelerators (World Scientific, Singapore, 1997).

[46] S. Arsenyev (private communication).

[47] M. J. Barnes, N. Garrel, T. Kroyer, F. Caspers, and L. Ducimetière, The beam screen for the LHC injection kicker magnets, in 10th European Particle Accelerator Conference (EPAC'06), Edinburgh, United Kingdom, 26-30 May, June, European Particle Accelerator Conference No. 10 (JACoW, Geneva, Switzerland, 2006), pp. 1508-1512.

[48] Opera-SIMULIA by Dassault Systèmes, Operaelectromagnetic and electromechanical simulation, https: //www.3ds.com/.

[49] A. Adraktas, L. Ducimetière, and M. J. Barnes, Influence of conducting serigraphy upon field pulse shape of the SPS extraction kicker systems, in Proceedings of the 8th 
International Particle Accelerator Conference (IPAC'17), Copenhagen, Denmark, 14-18 May, 2017, International Particle Accelerator Conference No. 8 (JACoW, Geneva, Switzerland, 2017), pp. 3491-3494.

[50] M. J. Barnes, T. Stadlbauer, and T. Kramer, Calculation of metallization resistivity and thickness for MedAustron kickers, in Proceedings of the 2nd International Particle Accelerator Conference (IPAC'11), San Sebastian, Spain, 4-9 September, 2011, International Particle Accelerator Conference No. 2 (JACoW, Geneva, Switzerland, 2011), pp. 3412-3414.

[51] ThoughtCo. Official Website, Table of Electrical Resistivity and Conductivity, https://www.thoughtco.com/tableof-electrical-resistivity-conductivity-608499 (2019).

[52] K. L. Kaiser, Electromagnetic Compatibility Handbook (CRC press, Boca Raton, Florida, 2004).

[53] Opera Simulation Software Suite, Opera-2D Reference Suite, (2018), (Version 18R2).
[54] M. J. Barnes, Spice models optimized to accurately simulate frequency-dependent impedances, Personal Engineering \& Instrumentation News 13, 63 (1996).

[55] J. Holma, A pulse power modulator with extremely flat-top output pulses for the Compact Linear Collider at CERN, Ph.D. thesis, Aalto University (2015), https://cds.cern.ch/ record/2130258/files/CERN-THESIS-2014-359.pdf.

[56] Heraeus Electronics Official Website, Products and Solutions, http://www.heraeus-electronics.com (2019).

[57] Huber + Suhner Official Website, Radio Frequency Products, http://www.hubersuhner.com (2019).

[58] H. Day, M. J. Barnes, F. Caspers, E. Métral, and B. Salvant, Beam coupling impedance of the new beam screen of the LHC injection kicker magnets, in Proceedings of the 5th International Particle Accelerator Conference (IPAC'14), Dresden, June 15-20, 2014, International Particle Accelerator Conference No. 2 (JACoW, Geneva, Switzerland, 2014), pp. 1627-1629. 